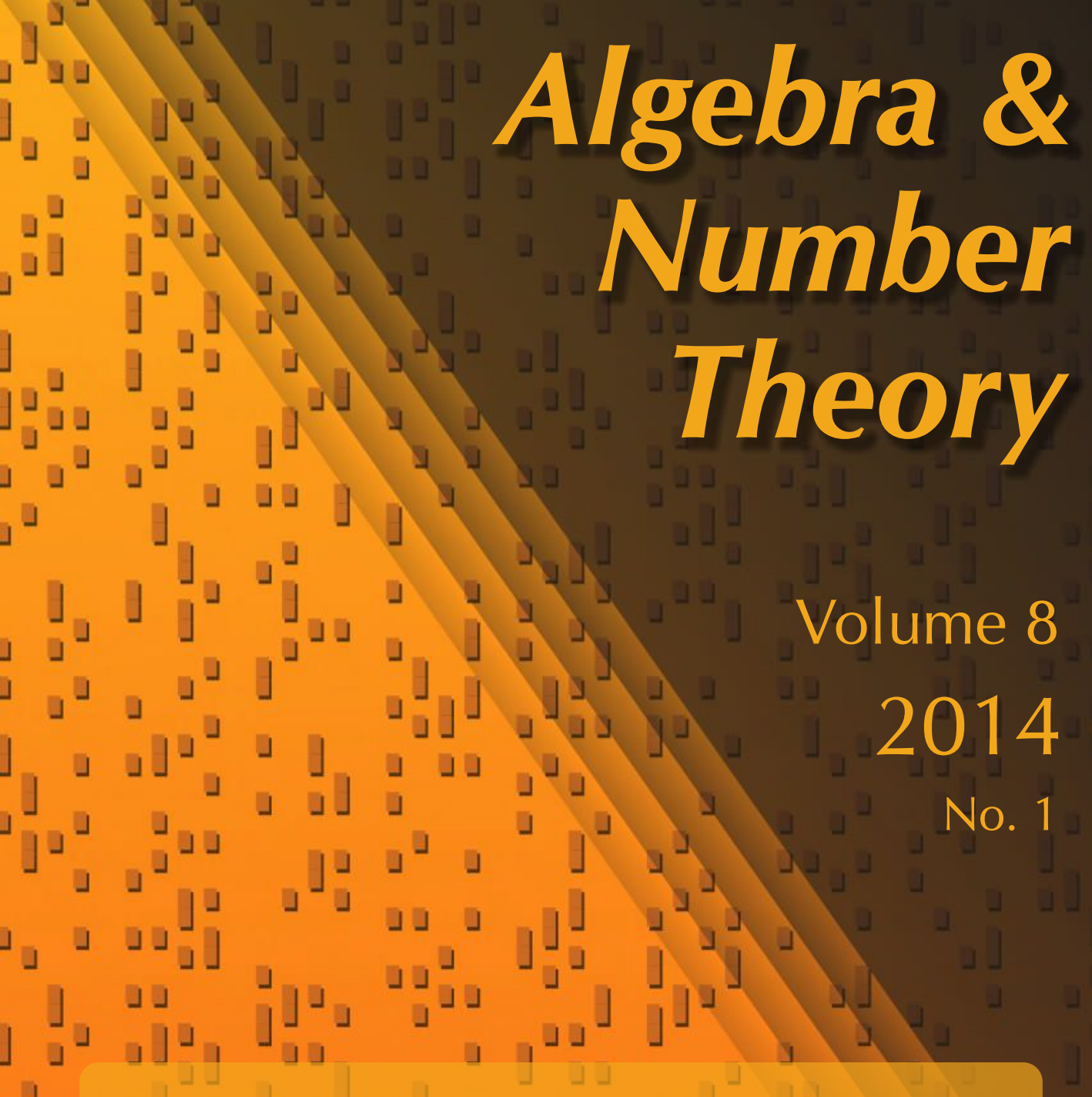

Adèle residue symbol and Tate's central extension

for multiloop Lie algebras

Oliver Braunling

\lrcorner

ل 


\title{
Adèle residue symbol and Tate's central extension for multiloop Lie algebras
}

\author{
Oliver Braunling
}

\begin{abstract}
We generalize the linear algebra setting of Tate's central extension to arbitrary dimension. In general, one obtains a Lie $(n+1)$-cocycle. We compute it to some extent. The construction is based on a Lie algebra variant of Beilinson's adelic multidimensional residue symbol, generalizing Tate's approach to the local residue symbol for 1 -forms on curves.
\end{abstract}

Firstly, recall that to every Lie algebra $\mathfrak{g}$ one can associate its loop Lie algebra $\mathfrak{g}\left[t^{ \pm}\right]$. Iterating this construction, we obtain multiloop Lie algebras $\mathfrak{g}\left[t_{1}^{ \pm 1}, \ldots, t_{n}^{ \pm 1}\right]$. To begin with, we show that various classes of interesting multiloop Lie algebras can all be embedded into a large (infinite-dimensional) Lie algebra:

Theorem 1. Let $k$ be a field and $n \geq 1$. There is a universal Lie algebra $\mathfrak{G}$ naturally containing, simultaneously,

(1) the abelian Lie algebra $k\left[t_{1}^{ \pm 1}, \ldots, t_{n}^{ \pm 1}\right]$,

(2) Lie algebras of derivations, e.g., spanned by

$$
t_{1}^{s_{1}} \cdots t_{n}^{s_{n}} \partial_{t_{i}} \quad\left(\text { acting on } k\left[t_{1}^{ \pm 1}, \ldots, t_{n}^{ \pm 1}\right]\right),
$$

(3) for any finite-dimensional simple Lie algebra $\mathfrak{g}$, the multiloop algebra

$$
\mathfrak{g}\left[t_{1}^{ \pm 1}, \ldots, t_{n}^{ \pm 1}\right]
$$

The universal Lie algebra $\mathfrak{G}$ has a canonical Lie $(n+1)$-cocycle $\phi \in H^{n+1}(\mathfrak{G}, k)$. For $n=1$ this cocycle determines a central extension

$$
0 \rightarrow k \rightarrow \widehat{\mathfrak{G}} \rightarrow \mathfrak{G} \rightarrow 0
$$

(known as Tate's central extension) and the pullback of it to one of the above types of subalgebras yields (respectively)

(1) the Heisenberg algebra,

This work has been supported by the DFG SFB/TR 45 "Periods, moduli spaces and arithmetic of algebraic varieties" and the Alexander von Humboldt Foundation.

MSC2010: primary 17B56, 17B67; secondary 32A27.

Keywords: adèle, residue symbol, Tate central extension, Kac-Moody, Japanese group. 
(2) the Virasoro algebra,

(3) the affine Lie algebra $\widehat{\mathfrak{g}}$ associated to $\mathfrak{g}$.

This will be stated in more detail and proven in Section 6. It is not at all surprising that some Lie algebras can be embedded into larger ones. The interesting fact is that there is such a Lie algebra which carries a canonical cocycle, inducing the ones defining all these classical central extensions. For $n=1$ the above is well-known see, for example, [Beilinson et al. 1991, §2.1]. For $n=1$, 2, see [Frenkel and Zhu 2012]. In the language of the latter, $\mathfrak{G}$ is an example of a "master Lie algebra".

We are interested in the nature of $\phi$ for $n>1$ - even if such cocycles cannot be interpreted as a central extension anymore (we get crossed modules, etc.). Indeed, they are meaningful, as we shall see.

A key point of this text is the actual computation of $\phi$ (with a slight limitation):

Theorem 2. The cocycle $\phi \in H^{n+1}(\mathfrak{G}, k)$ is given explicitly by

$\phi\left(f_{0} \wedge f_{1} \wedge \cdots \wedge f_{n}\right)$
$=\operatorname{tr} \sum_{\pi \in \mathfrak{S}_{n}} \operatorname{sgn} \pi \sum_{\gamma_{1}, \ldots, \gamma_{n} \in\{ \pm\}}(-1)^{\gamma_{1}+\cdots+\gamma_{n}}\left(P_{1}^{-\gamma_{1}} \operatorname{ad}\left(f_{\pi(1)}\right) P_{1}^{\gamma_{1}}\right) \cdots\left(P_{n}^{-\gamma_{n}} \operatorname{ad}\left(f_{\pi(n)}\right) P_{n}^{\gamma_{n}}\right) f_{0}$,

whenever $f_{0} \otimes f_{1} \wedge \cdots \wedge f_{n}$ is already a $\mathfrak{g}$-valued Lie cycle. The $P_{1}^{+}, \ldots, P_{n}^{+}$refer to certain commuting idempotents (see Section 4 for details).

The proof and details regarding the $P_{i}^{ \pm}$can be found in Section 6. Effectively, we compute the composition

$$
H_{n}(\mathfrak{g}, \mathfrak{g}) \stackrel{I}{\rightarrow} H_{n+1}(\mathfrak{g}, k) \rightarrow k,
$$

with $I$ a natural map to be explained in Section 2. By the universal coefficient theorem for Lie algebras, $H^{n+1}(\mathfrak{g}, k) \cong H_{n+1}(\mathfrak{g}, k)^{*}$, referring to the dual space. As such, although $\phi$ is well-defined, the formula only applies to those cycles admitting a lift under $I$ (as soon as it exists, the choice does not matter). The formula is rather complicated. However, the pullback to particular subalgebras of $\mathfrak{G}$ can be much nicer; for example for multiloop Lie algebras of simple Lie algebras, we get the following:

Theorem 3. Suppose $\mathfrak{g} / k$ is a finite-dimensional centerless Lie algebra (e.g., simple). For $Y_{0}, \ldots, Y_{n} \in \mathfrak{g}$ we call

$$
B\left(Y_{0}, \ldots, Y_{n}\right):=\operatorname{tr}_{\operatorname{End}_{k}(\mathfrak{g})}\left(\operatorname{ad}\left(Y_{0}\right) \operatorname{ad}\left(Y_{1}\right) \cdots \operatorname{ad}\left(Y_{n}\right)\right)
$$


the "generalized Killing form". Then on all Lie cycles admitting a lift under I as in (0-1), the pullback of $\phi$ to $\mathfrak{g}\left[t_{1}^{ \pm}, \ldots, t_{n}^{ \pm}\right]$is explicitly given by

$$
\begin{aligned}
\phi\left(Y_{0} t_{1}^{c_{0,1}} \cdots t_{n}^{c_{0, n}} \wedge \cdots \wedge Y_{n} t_{1}^{c_{n, 1}} \cdots t_{n}^{c_{n, n}}\right) \\
=(-1)^{n} \sum_{\pi \in \mathfrak{S}_{n}} \operatorname{sgn} \pi B\left(Y_{\pi(1)}, \ldots, Y_{\pi(n)}, Y_{0}\right) \prod_{i=1}^{n} c_{\pi(i), i}
\end{aligned}
$$

whenever $\sum_{p=0}^{n} c_{p, i}=0$ for all $i \in\{1, \ldots, n\}$, and vanishes otherwise. Here $c_{i, p} \in \mathbb{Z}$ for all $i=0, \ldots, n$ and $p=1, \ldots, n$.

If $\mathfrak{g}$ is finite-dimensional simple and $n=1$, then the class $\phi$ yields the universal central extension of the loop Lie algebra $\mathfrak{g}\left[t_{1}, t_{1}^{-1}\right]$, the associated affine Lie algebra $\widehat{\mathfrak{g}}$ (without extending by a derivation),

$$
0 \rightarrow k \rightarrow \widehat{\mathfrak{g}} \rightarrow \mathfrak{g}\left[t_{1}, t_{1}^{-1}\right] \rightarrow 0 .
$$

In this case $B$ is obviously just the ordinary Killing form of $\mathfrak{g}$. The above theorem will be proven in Section 8 .

Additionally, we should say that these computations have an application outside the theory of Lie algebras. For this we need to return to the roots of the subject. J. Tate [1968] showed that the residue of a rational 1-form $f \mathrm{~d} g$ at a closed point $x$ on an algebraic curve $X / k$ can be expressed as a certain operator-theoretic trace on an infinite-dimensional space. Arbarello, de Concini and Kac [Arbarello et al. 1989, eq. (2.7)] reformulated this as

$$
\operatorname{res}_{x} f \mathrm{~d} g=\operatorname{tr}([\pi, g] f) .
$$

On the right-hand side the functions $f, g$ are to be read as multiplication operators acting on the local field Frac $\widehat{\widehat{O}}_{X, x} \simeq \kappa(x)\left(\left(t_{1}\right)\right)$, seen as a $\kappa(x)$-vector space, and $\pi$ denotes some projector on the nonprincipal part, i.e., "we cut off the principal part of the Laurent series." It is natural to ask whether there exists a generalization of this formula to higher residues. We can give such a formula; it will be proven in Section 7:

Theorem 4. For a multiple Laurent polynomial ring with residue field $k$, say

$$
R:=k\left[t_{1}^{ \pm}, \ldots, t_{n}^{ \pm}\right]
$$

and $f_{0}, \ldots, f_{n} \in R$ we have

$$
\begin{aligned}
& \operatorname{res}_{t_{1}} \ldots \operatorname{res}_{t_{n}} f_{0} \mathrm{~d} f_{1} \ldots \mathrm{d} f_{n} \\
& =(-1)^{n} \operatorname{tr} \sum_{\pi \in \mathfrak{S}_{n}} \operatorname{sgn} \pi \sum_{\gamma_{1} \ldots \gamma_{n} \in\{ \pm\}}(-1)^{\gamma_{1}+\cdots+\gamma_{n}} \\
& \quad \times\left(P_{1}^{-\gamma_{1}} \operatorname{ad}\left(f_{\pi(1)}\right) P_{1}^{\gamma_{1}}\right) \cdots\left(P_{n}^{-\gamma_{n}} \operatorname{ad}\left(f_{\pi(n)}\right) P_{n}^{\gamma_{n}}\right) f_{0},
\end{aligned}
$$


where $P_{1}^{ \pm}, \ldots, P_{n}^{ \pm}$are suitable projectors (explained in Section 7; see (7-3)).

(1) For $n=1$ and $\pi:=P_{1}^{+}$the formula reduces to the familiar (0-2) (as in [Arbarello et al. 1989]).

(2) If we have $f_{i}=t_{1}^{c_{i, 1}} \cdots t_{n}^{c_{i, n}}$ for $i=0, \ldots, n$, the formula reduces to

$$
\operatorname{res} f_{0} \mathrm{~d} f_{1} \cdots \mathrm{d} f_{n}=\operatorname{det}\left(\begin{array}{ccc}
c_{1,1} & \cdots & c_{n, 1} \\
\vdots & \ddots & \vdots \\
c_{1, n} & \cdots & c_{n, n}
\end{array}\right) \quad \text { if } \sum_{p=0}^{n} c_{p, i}=0 \text { for all } i
$$

and the residue is zero if the condition on the right-hand side is not satisfied.

(3) For $n=1$ and $f_{1}=t_{1}$ this reduces by linearity to the classical definition

$$
\operatorname{res} \alpha t_{1}^{c_{1}} \mathrm{~d} t_{1}= \begin{cases}\alpha & \text { if } c_{1}=-1 \\ 0 & \text { if } c_{1} \neq-1\end{cases}
$$

How do we construct the cocycle $\phi$ ?

There are various ways to approach this construction. Frenkel and Zhu [2012] use distinguished generators of the cohomology ring of infinite matrix algebras, based on computations of Feigin and Tsygan [1983]. This is a very natural approach. However, in this text we use a different approach based on the multidimensional adelic residue of [Beilinson 1980]. Originally, this approach was only used to generalize Tate's approach to the residue symbol to several variables, but it readily generalizes to the problem we are discussing here. This might be interesting also since Beilinson does not give an explicit formula - and it is not totally trivial to extrapolate a formula from the definition.

Theorem 5. The formula in Theorem 4 arises from the construction of Beilinson (in Lemma 1 of [Beilinson 1980]), i.e., it is the composition

$$
\Omega_{R / k}^{n} \stackrel{(-1)^{n} \varkappa}{\longrightarrow} H_{n+1}^{\mathrm{Lie}}(\mathfrak{G}, k) \stackrel{\rho_{2}}{\rightarrow} E_{0, n+1}^{n+1} \stackrel{\left(d_{n+1}\right)^{-1}}{\longrightarrow} \wedge E_{n+1,1}^{n+1} \stackrel{\rho_{1}}{\rightarrow} H_{0}^{\mathrm{Lie}}\left(\mathfrak{G}, N^{n+1}\right) \stackrel{\operatorname{tr}}{\rightarrow} k,
$$

where

- $\varkappa: f_{0} \mathrm{~d} f_{1} \wedge \cdots \wedge \mathrm{d} f_{n} \mapsto f_{0} \wedge \cdots \wedge f_{n}$,

- $N^{n+1}$ is a certain $\mathfrak{G}$-module (see Section 4 for the definition, or $T_{* N}$ in [Beilinson 1980]), and

- $\rho_{1}, \rho_{2}$ are edge maps and $d_{n+1}$ a differential on the $(n+1)$-st page of a certain spectral sequence ${ }^{\wedge} E_{\bullet, \bullet}^{\bullet}$ (constructed in Lemma 19, or see [Beilinson 1980, Lemma 1]).

This result is only meaningful to readers familiar with [Beilinson 1980]. 
The above theorem actually lies at the heart of our approach. We formulate a contracting homotopy for a mild variation of the relevant complexes in [Beilinson 1980] and then, in a slightly tedious computation, make the spectral sequence differential $d_{n+1}$ explicit on the basis of this.

Finally, for applications in algebraic geometry, e.g., the interpretation as a local residue, it is unfortunate to interpret "loop Lie algebra" as $\mathfrak{g}\left[t, t^{-1}\right]$. It is better to work with Laurent series, i.e., $\mathfrak{g}((t))$, or even local components of adèles. Tate's original work uses the language of adèles for example. For this reason, we shall axiomatize all these variations through the notion of a "cubically decomposed algebra" (essentially taken from [Beilinson 1980], where it's not given a name).

What is not here. In the present text I only discuss the "linear algebra setting" of Tate's central extension ([Beilinson et al. 1991, §1] for the case $n=1$ ). There is also a "differential operator setting" [ibid., §2], which I will treat in a future text. Roughly speaking, $\mathfrak{G}$ will be replaced by much smaller algebras of differential operators on a vector bundle.

Moreover, I do not treat the true multiloop analogue of an affine Kac-Moody algebra in the present text. Already for $n=1 \mathrm{I}$ only consider the "plain" affine Lie algebras without extending by a derivation. From the perspective of a triangular decomposition, this is a rather horrible omission: the root spaces are infinitedimensional! However, as the reader can probably imagine from the computations in Sections 7 and 8 the calculation gets a lot more complicated in the presence of derivations. Thus, this aspect will also be deferred to a future text. The same applies to the analogue of the plain Virasoro algebra. There should also be a nonlinear analogue, distinguished cohomology classes for multiloop groups. The cases $n=1,2$ (along with a higher representation theory in categories) are treated in detail by Frenkel and Zhu [2012].

One should also mention that there are completely orthogonal generalizations of Kac-Moody/Virasoro cocycles to multiloop Lie algebras - see, for example, [Frenkel 1987, §9; Neher 2011].

\section{Basic framework}

For an associative algebra $A$ we shall write $A_{\text {Lie }}$ to denote the associated Lie algebra.

Definition 6 [Beilinson 1980]. An (n-fold) cubically decomposed algebra (over a field $k)$ is the datum $\left(A,\left(I_{i}^{ \pm}\right), \tau\right)$ :

- an associative unital (not necessarily commutative) $k$-algebra $A$;

- two-sided ideals $I_{i}^{+}, I_{i}^{-}$such that $I_{i}^{+}+I_{i}^{-}=A$ for $i=1, \ldots, n$; 
- writing $I_{i}^{0}:=I_{i}^{+} \cap I_{i}^{-}$and $I_{\mathrm{tr}}:=I_{1}^{0} \cap \cdots \cap I_{n}^{0}$, a $k$-linear map

$$
\tau: I_{\mathrm{tr}, \mathrm{Lie}} /\left[I_{\mathrm{tr}, \mathrm{Lie}}, A_{\text {Lie }}\right] \rightarrow k .
$$

For any finite-dimensional $k$-vector space $V$, certain infinite matrix algebras act naturally on the $k$-vector space of multiple Laurent polynomials $V\left[t_{1}^{ \pm 1}, \ldots, t_{n}^{ \pm 1}\right]$. This yields an example of this structure - see Section 1.1. There is also an analogue for $V\left(\left(t_{1}\right)\right) \cdots\left(\left(t_{n}\right)\right)$, which we leave to the reader to formulate (this links to higher local fields, see [Fesenko and Kurihara 2000]). Local components of ParshinBeilinson adèles of schemes yield another example, see [Beilinson 1980, §1]. In loc. cit. the ideals $I_{i}^{+}, I_{i}^{-}$are called $X^{i}, Y^{i}$. The latter gives the multidimensional generalization of the adèle formulation of Tate [1968]. See [Fesenko 2010; Huber 1991; Hübl and Yekutieli 1996; Morrow 2010] for more background on higherdimensional adèles and their uses.

1.1. Infinite matrix algebras. Fix a field $k$. Let $R$ be an associative $k$-algebra, not necessarily unital or commutative. Define an algebra of infinite matrices

$$
E(R):=\left\{\phi=\left(\phi_{i j}\right)_{i, j \in \mathbb{Z}}, \phi_{i j} \in R\left|\exists K_{\phi}:\right| i-j \mid>K_{\phi} \Rightarrow \phi_{i j}=0\right\} .
$$

Define a product by $\left(\phi \cdot \phi^{\prime}\right)_{i k}:=\sum_{j \in \mathbb{Z}} \phi_{i j} \phi_{j k}^{\prime}$, the usual matrix multiplication formula; this sum only has finitely many nonzero terms and one can choose $K_{\phi \phi^{\prime}}:=$ $K_{\phi}+K_{\phi^{\prime}}$. Then $E(R)$ becomes an associative $k$-algebra. If $R$ is unital, $E(R)$ is also unital. $E$ is a functor from associative algebras to associative algebras; for a morphism $\varphi: R \rightarrow S$ there is an induced morphism $E(\varphi): E(R) \rightarrow E(S)$ by using $\varphi$ entry-by-entry, i.e., $(E(\varphi) \phi)_{i j}:=\varphi\left(\phi_{i j}\right)$. If $I \subseteq R$ is an ideal (which is in particular a nonunital associative ring), $E(I) \subseteq E(R)$ is an ideal. Moreover, for ideals $I_{1}, I_{2}$ one has $E\left(I_{1} \cap I_{2}\right)=E\left(I_{1}\right) \cap E\left(I_{2}\right)$ and $E\left(I_{1}+I_{2}\right)=E\left(I_{1}\right)+E\left(I_{2}\right)$, as a sum of ideals. Next, define

$$
\begin{aligned}
& I^{+}(R):=\left\{\phi \in E(R) \mid \exists B_{\phi}: i<B_{\phi} \Rightarrow \phi_{i j}=0\right\}, \\
& I^{-}(R):=\left\{\phi \in E(R) \mid \exists B_{\phi}: j>B_{\phi} \Rightarrow \phi_{i j}=0\right\}
\end{aligned}
$$

and one checks easily that $I^{+}(R), I^{-}(R)$ are two-sided ideals in $E(R)$. The following figure attempts to visualize the shape of the matrices in $E(R), I^{+}(R)$ and $I^{-}(R)$, respectively:

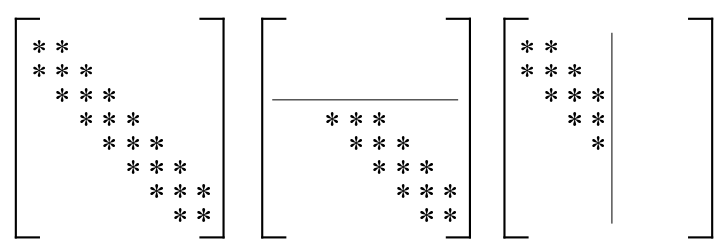


Define $I^{0}(R):=I^{+}(R) \cap I^{-}(R)$ and one checks that

$$
I^{0}(R):=\left\{\phi \in E(R) \mid \phi_{i j}=0 \text { for all but finitely many }(i, j)\right\} .
$$

There is a trace morphism

$$
\operatorname{tr}: I^{0}(R) \rightarrow R, \quad \operatorname{tr} \phi:=\sum_{i \in \mathbb{Z}} \phi_{i i}
$$

the sum is obviously finite. One easily verifies that $\operatorname{tr}\left[\phi, \phi^{\prime}\right]=\sum_{i, j \in \mathbb{Z}}\left[\phi_{i j}, \phi_{j i}^{\prime}\right]$ and thus $\operatorname{tr}\left[I^{0}(R), E(R)\right] \subseteq[R, R]$. More generally, if $R^{\prime} \subseteq R$ is a subalgebra,

$$
\operatorname{tr}\left[I^{0}\left(R^{\prime}\right), E(R)\right] \subseteq\left[R^{\prime}, R\right]
$$

We note that this trace does not necessarily vanish on commutators. Moreover, every $\phi \in E(R)$ can be written as $\phi=\phi^{+}+\phi^{-}$with $\phi_{i j}^{+}:=\delta_{i \geq 0} \phi_{i j}$ (for this $R$ need not be unital, use $\phi_{i j}$ for $i \geq 0$ and 0 otherwise) and $\phi^{-}=\phi-\phi^{+}$. One checks that $\phi^{ \pm} \in I^{ \pm}(R)$. It follows that $I^{+}(R)+I^{-}(R)=E(R)$.

Finally, let $M$ be an $R$-bimodule (over $k$, i.e., a left- $\left(A \otimes_{k} A^{o p}\right)$-module; $R$ bimodules form an abelian category). Analogously to $E(R)$, define

$$
E(M):=\left\{\phi=\left(\phi_{i j}\right)_{i, j \in \mathbb{Z}}, \phi_{i j} \in M\left|\exists K_{\phi}:\right| i-j \mid>K_{\phi} \Rightarrow \phi_{i j}=0\right\} .
$$

Again using the matrix multiplication formula, $E(M)$ is an $E(R)$-bimodule. If $0 \rightarrow M^{\prime} \rightarrow M \rightarrow M^{\prime \prime} \rightarrow 0$ is an exact sequence of $R$-bimodules, $0 \rightarrow E\left(M^{\prime}\right) \rightarrow$ $E(M) \rightarrow E\left(M^{\prime \prime}\right) \rightarrow 0$ is an exact sequence of $E(R)$-bimodules. Note that for an ideal $I \subseteq R$ the object $E(I)$ is well-defined, regardless of whether we regard $I$ as an associative ring as in (1-1) or an $R$-bimodule as in (1-3).

Now let $V$ be a finite-dimensional $k$-vector space and $R_{0}$ an arbitrary unital subalgebra of $\operatorname{End}_{k}(V)$. Define $R_{i}:=E\left(R_{i-1}\right)$ for $i=1, \ldots, n$. Note that via $k \rightarrow R_{0}, \alpha \mapsto \alpha \cdot \mathbb{1}_{\operatorname{End}_{k}(V)}, k$ is embedded into the center of $R_{i}$. Then $R_{n}=$ $(E \circ \cdots \circ E)\left(R_{0}\right)$ is a unital associative $k$-algebra. Its elements may be indexed

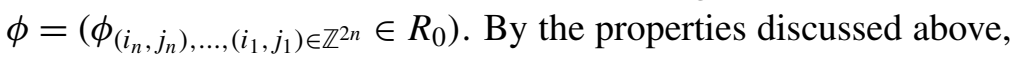

$$
I_{i}^{ \pm}:=\left(\underset{n}{E} \cdots \underset{i+1}{E} \circ I_{i}^{ \pm} \circ \underset{i-1}{E} \cdots \underset{1}{E}\right)\left(R_{0}\right) \quad\left(I^{ \pm} \text {in the } i \text {-th place }\right)
$$

is an ideal in $R_{n}$ (we use centered subscripts only to emphasize the numbering). Moreover,

$$
\begin{aligned}
I_{i}^{+}+I_{i}^{-} & =\left(E \cdots E \circ I^{+} \circ E \cdots E\right)\left(R_{0}\right)+\left(E \cdots E \circ I^{-} \circ E \cdots E\right)\left(R_{0}\right) \\
& =(E \cdots E \circ E \circ E \cdots E)\left(R_{0}\right)=R_{n} .
\end{aligned}
$$


By composing the traces of (1-2) we arrive at a $k$-linear map $\tau$,

$$
\begin{aligned}
\tau: I_{\mathrm{tr}} & =I_{1}^{0} \cap \cdots \cap I_{n}^{0} \\
& =\left(I^{0} \circ \cdots \circ I^{0}\right)\left(R_{0}\right) \stackrel{\operatorname{tr}}{\rightarrow} \cdots \stackrel{\operatorname{tr}}{\rightarrow} I^{0}\left(I^{0}\left(R_{0}\right)\right) \stackrel{\text { tr }}{\rightarrow} I^{0}\left(R_{0}\right) \stackrel{\operatorname{tr}}{\rightarrow} R_{0} \stackrel{\operatorname{Tr}}{\rightarrow} k,
\end{aligned}
$$

where "Tr" (as opposed to "tr") denotes the ordinary matrix trace of $\operatorname{End}_{k}(V)$ $\left(\supseteq R_{0}\right)$. Here we have used that $V$ is finite-dimensional over $k$. Using inductively the relation

$$
\operatorname{tr}\left[I^{0}\left(R^{\prime}\right), E(R)\right] \subseteq\left[R^{\prime}, R\right]
$$

(valid for subalgebras $R^{\prime} \subseteq R$ ), one sees that

$$
\begin{aligned}
\tau\left[I_{\mathrm{tr}}, R_{n}\right] & =\operatorname{Tr}(\operatorname{tr} \circ \cdots \circ \operatorname{tr} \circ \operatorname{tr})\left[I^{0}\left(I^{0}(\cdots)\right), E(E(\cdots))\right] \\
& \subseteq \operatorname{Tr}(\operatorname{tr} \circ \cdots \circ \operatorname{tr})\left[I^{0}(\cdots), E(\cdots)\right] \subseteq \operatorname{Tr}\left[R_{0}, R_{0}\right]=0
\end{aligned}
$$

since the ordinary trace $\operatorname{Tr}$ vanishes on commutators. Hence, $\tau$ factors to a morphism $\tau: I_{\mathrm{tr}, \mathrm{Lie}} /\left[I_{\mathrm{tr}, \mathrm{Lie}}, R_{\mathrm{Lie}}\right] \rightarrow k$. Summarizing, for every $n \geq 1$, every finite-dimensional $k$-vector space $V$ and every unital subalgebra $R_{0} \subseteq \operatorname{End}_{k}(V),\left(R_{n},\left(I_{i}^{ \pm}\right), \tau\right)$ is a cubically decomposed algebra.

Finally, note that for any associative algebra $R, E(R)$ is a right- $R$-submodule of right-R-module endomorphisms $\operatorname{End}_{R}\left(R\left[t, t^{-1}\right]\right)$ of $R\left[t, t^{-1}\right]$. Write elements as $a=\sum_{i \in \mathbb{Z}} a_{i} t^{i}$, also denoted $a=\left(a_{i}\right)_{i}$ with $a_{i} \in R$, and let $\phi=\left(\phi_{i j}\right)$ act by $(\phi \cdot a)_{i}:=\sum_{k} \phi_{i k} a_{k}$. Moreover, each $a \in R\left[t, t^{-1}\right]$ determines a right- $R$-module endomorphism via the multiplication operator $x \mapsto a \cdot x$. We find

$$
R\left[t, t^{-1}\right] \hookrightarrow E(R) \hookrightarrow \operatorname{End}_{R}\left(R\left[t, t^{-1}\right]\right) .
$$

Multiplication with $t^{i}$ is represented by a matrix with a diagonal $\ldots, 1,1,1, \ldots$, shifted by $i$ off the principal diagonal. Inductively,

$$
R_{0}\left[t_{1}^{ \pm 1}, \ldots, t_{n}^{ \pm 1}\right] \hookrightarrow R_{n} \hookrightarrow \operatorname{End}_{R_{0}}\left(R_{0}\left[t_{1}^{ \pm 1}, \ldots, t_{n}^{ \pm 1}\right]\right)
$$

See for example [Jimbo and Miwa 1983, §1; Kac and Raina 1987, Lec. 4] for more information regarding the case $n=1$ and [Frenkel and Zhu 2012, §3] for a similar procedure when $n=2$.

\section{Modified Chevalley-Eilenberg complexes}

Suppose $k$ is a field and $\mathfrak{g}$ a Lie algebra over $k$. We recall that for any $\mathfrak{g}$-module the conventional Chevalley-Eilenberg complex is given by $C(M)_{r}:=M \otimes \wedge^{r} \mathfrak{g}$ along 
with the differential

$$
\begin{aligned}
\delta & :=\delta^{[1]}+\delta^{[2]}: C(M)_{r} \rightarrow C(M)_{r-1}, \\
\delta^{[1]}\left(f_{0} \otimes f_{1} \wedge \cdots \wedge f_{r}\right) & :=\sum_{i=1}^{r}(-1)^{i}\left[f_{0}, f_{i}\right] \otimes f_{1} \wedge \cdots \wedge \widehat{f_{i}} \wedge \cdots \wedge f_{r}, \\
\delta^{[2]}\left(f_{0} \otimes f_{1} \wedge \cdots \wedge f_{r}\right) & :=\sum_{1 \leq i<j \leq r}(-1)^{i+j+1} f_{0} \otimes\left[f_{i}, f_{j}\right] \wedge f_{1} \wedge \cdots \widehat{f_{i}} \cdots \widehat{f_{j}} \cdots \wedge f_{r}
\end{aligned}
$$

for $f_{0} \in M$ and $f_{1}, \ldots, f_{r} \in \mathfrak{g}$. Its homology is (by definition, if one wants) Lie homology with coefficients in $M$. There is also a cohomological analogue; for details see, for example, [Loday 1992, Chapter 10]. We may view $k$ itself as a $\mathfrak{g}$-module with the trivial structure. There is an obvious morphism

$$
I: C(\mathfrak{g})_{r} \rightarrow C(k)_{r+1}, \quad f_{0} \otimes f_{1} \wedge \cdots \wedge f_{r} \mapsto(-1)^{r} \mathbb{1}_{k} \otimes f_{0} \wedge f_{1} \wedge \cdots \wedge f_{r},
$$

and one checks easily that this commutes with the respective differentials and thus induces morphisms $H_{r}(\mathfrak{g}, \mathfrak{g}) \rightarrow H_{r+1}(\mathfrak{g}, k)$. The linear dual $\mathfrak{g}^{*}:=\operatorname{Hom}_{k}(\mathfrak{g}, k)$ is canonically a $\mathfrak{g}$-module via $(f \cdot \varphi)(g):=\varphi([g, f])$ for $\varphi \in \mathfrak{g}^{*}$ and $f, g \in \mathfrak{g}$. The cohomological analogue of (2-2) is the morphism $I: H^{r+1}(\mathfrak{g}, k) \rightarrow H^{r}\left(\mathfrak{g}, \mathfrak{g}^{*}\right)$ given by

$$
(I \phi)\left(f_{1} \wedge \cdots \wedge f_{r}\right)\left(f_{0}\right):=(-1)^{r} \phi\left(f_{0} \wedge f_{1} \wedge \cdots \wedge f_{r}\right) .
$$

Remark 7. These maps could be viewed as a Lie-theoretic analogue of map $I$ in Connes' periodicity sequence - see [Loday 1992, §2.2]. We may view $H_{*-1}(\mathfrak{g}, \mathfrak{g})$ as a partial "noncyclic" counterpart of Lie homology. The true Hochschild analogue would be Leibniz homology — see [Loday 1992, §10.6]. For the present purposes, however, we have no use for this analogue.

Let $\mathfrak{j} \subseteq \mathfrak{g}$ be a Lie ideal. As such, it is a $\mathfrak{g}$-module and we may consider $C(\mathrm{j})$. Following [Beilinson 1980] we may work with a "cyclically symmetrized" counterpart: We write $\mathfrak{j} \wedge \wedge^{r-1} \mathfrak{g}$ to denote the $\mathfrak{g}$-submodule of $\mathfrak{g} \wedge \wedge^{r-1} \mathfrak{g}=\bigwedge^{r} \mathfrak{g}$ generated by elements $j \wedge f_{1} \wedge \cdots \wedge f_{r-1}$ such that $j \in \mathfrak{j}$ and $f_{1}, \ldots, f_{r-1} \in \mathfrak{g}$. If $\mathfrak{j}_{i}, i=1,2, \ldots$, are Lie ideals, we denote by $\left(\bigoplus_{i} \mathfrak{j}_{i}\right) \wedge \wedge^{r-1} \mathfrak{g}$ the module $\bigoplus_{i}\left(\mathfrak{j}_{i} \wedge \wedge^{r-1} \mathfrak{g}\right)$.

Example 8. If $k\langle s, t, u\rangle$ and $k\langle s\rangle$ denote a 3-dimensional abelian Lie algebra along with a 1-dimensional Lie ideal, then $\wedge^{2} k\langle s, t, u\rangle$ is 3-dimensional with basis $s \wedge t$, $s \wedge u$ and $t \wedge u$. Then $k\langle s\rangle \wedge k\langle s, t, u\rangle$ is 2-dimensional with basis $s \wedge t, s \wedge u$.

The $k$-vector spaces $C E(\mathfrak{j})_{r}:=\mathfrak{j} \wedge \wedge^{r-1} \mathfrak{g}$ (for $\left.r \geq 1\right)$ and $C E(\mathfrak{j})_{0}:=k$ define a subcomplex of $C(k)$.. In particular, the differential is given by

$$
\delta\left(f_{0} \wedge f_{1} \wedge \cdots \wedge f_{r}\right):=\sum_{0 \leq i<j \leq r}(-1)^{i+j}\left[f_{i}, f_{j}\right] \wedge f_{0} \wedge \cdots \widehat{f_{i}} \cdots \widehat{f_{j}} \cdots \wedge f_{r}
$$


It is well-defined since $\mathrm{j}$ is a Lie ideal. We get morphisms generalizing $I$, notably $H_{r}(\mathfrak{g}, \mathfrak{j}) \rightarrow H_{r+1}(C E(\mathfrak{j}))$ via $\mathfrak{j} \otimes \wedge^{r} \mathfrak{g} \rightarrow \mathfrak{j} \wedge \wedge^{r} \mathfrak{g}$ and analogously $H^{r+1}(C E(\mathfrak{j})) \rightarrow$ $H^{r}\left(\mathfrak{g}, \mathfrak{j}^{*}\right)$. We have resisted the temptation to reindex $C E(-)$. despite the unpleasant $(+1)$-shift in (2-2) in order to remain compatible with standard usage in the following sense:

Lemma 9 [Beilinson 1980, Lemma 1(a)]. CE(g). is a complex of $k$-vector spaces and is quasi-isomorphic to $k \otimes_{U \mathfrak{g}}^{\mathbf{L}} k$. In particular

$$
H_{i}(\mathfrak{g}, k)=H_{i}(C E(\mathfrak{g}) .) \quad \text { and } \quad H^{i}(\mathfrak{g}, k)=H^{i}\left(\operatorname{Hom}_{k}\left(C E(\mathfrak{g})_{\bullet}, k\right)\right) .
$$

Proof. As we have explained above, $C E(\mathfrak{g})$. agrees with the standard ChevalleyEilenberg complex and the latter is well-known to represent $k \otimes_{U \mathfrak{g}}^{\mathbf{L}} k$.

We easily compute

$$
\begin{aligned}
H_{0}(\mathfrak{g}, \mathfrak{j}) & \stackrel{\cong}{\longrightarrow} H_{1}(C E(\mathfrak{j})) \cong \mathfrak{j} /[\mathfrak{g}, \mathfrak{j}], \\
H^{1}(C E(\mathfrak{j})) & \stackrel{\cong}{I} H^{0}\left(\mathfrak{g}, \mathfrak{j}^{*}\right) \cong(\mathfrak{j} /[\mathfrak{g}, \mathfrak{j}])^{*} .
\end{aligned}
$$

In higher degrees the map $I$ ceases to be an isomorphism.

Nonetheless, this computation hints at the principle of computation which we shall use below. Beilinson [1980] uses $C E(-)$., whereas we will only be able to do manageable computations with $C(-)$. The map $I$ will serve to deduce facts about $C E(-)$. while working with $C(-)$.

\section{Cubically decomposed algebras}

Let $\left(A,\left(I_{i}^{ \pm}\right), \tau\right)$ be an $n$-fold cubically decomposed algebra (Definition 6) over a field $k$; that is, we are given the following data:

- an associative unital (not necessarily commutative) $k$-algebra $A$;

- two-sided ideals $I_{i}^{+}, I_{i}^{-}$such that $I_{i}^{+}+I_{i}^{-}=A$ for $i=1, \ldots, n$;

- writing $I_{i}^{0}:=I_{i}^{+} \cap I_{i}^{-}$and $I_{\mathrm{tr}}:=I_{1}^{0} \cap \cdots \cap I_{n}^{0}$, a $k$-linear map

$$
\tau: I_{\text {tr }, \text { Lie }} /\left[I_{\text {tr }, \text { Lie }}, A_{\text {Lie }}\right] \rightarrow k .
$$

See Section 1 to see how this type of structure arises. As a shorthand, define $\mathfrak{g}:=A_{\text {Lie }}$. For any elements $s_{1}, \ldots, s_{n} \in\{+,-, 0\}$ we define the degree of the $n$-tuple $\left(s_{1}, \ldots, s_{n}\right)$ as

$$
\operatorname{deg}\left(s_{1} \ldots s_{n}\right):=1+\#\left\{i \mid s_{i}=0\right\} .
$$

Next, following [Beilinson 1980], we construct complexes of $\mathfrak{g}$-modules: 
Definition 10 [Beilinson 1980]. For every $1 \leq p \leq n+1$ define

$$
{ }^{\wedge} T_{\bullet}^{p}:=\coprod_{\substack{s_{1} \ldots s_{n} \in\{ \pm, 0\} \\ \operatorname{deg}\left(s_{1} \ldots s_{n}\right)=p}} \bigcap_{i=1}^{n} \begin{cases}C E\left(I_{i}^{+}\right) . & \text {for } s_{i}=+, \\ C E\left(I_{i}^{-}\right) . & \text {for } s_{i}=-, \\ C E\left(I_{i}^{+}\right) . \cap C E\left(I_{i}^{-}\right) . & \text {for } s_{i}=0,\end{cases}
$$

and ${ }^{\wedge} T_{\bullet}^{0}:=C E(\mathfrak{g})$.

Each $C E\left(I_{i}^{ \pm}\right)$. is a complex and all their differentials are defined by the same formula, (2-3); hence the intersection of these complexes has a well-defined differential and is a complex itself. Same for the coproduct. The complex ${ }^{\wedge} T_{\bullet}^{\bullet}$ is inspired by a cubical object used by Beilinson [1980].

Example 11. For $n=2$ we get complexes

$$
\begin{aligned}
& { }^{\wedge} T_{\bullet}^{1}=\coprod_{s_{1}, s_{2} \in\{ \pm\}} C E\left(I_{1}^{s_{1}}\right) \cdot \cap C E\left(I_{2}^{s_{2}}\right) ., \\
& { }^{\wedge} T_{\bullet}^{2}=\coprod_{s_{1} \in\{ \pm\}} C E\left(I_{1}^{s_{1}}\right) . \cap C E\left(I_{2}^{+}\right) \cdot \cap C E\left(I_{2}^{-}\right) . \oplus \coprod_{s_{2} \in\{ \pm\}} C E\left(I_{1}^{+}\right) \cdot \cap C E\left(I_{1}^{-}\right) . \cap C E\left(I_{2}^{s_{2}}\right) ., \\
& { }^{\wedge} T_{\bullet}^{3}=C E\left(I_{1}^{+}\right) . \cap C E\left(I_{1}^{-}\right) . \cap C E\left(I_{2}^{+}\right) . \cap C E\left(I_{2}^{-}\right) . \bullet
\end{aligned}
$$

Note that $C E\left(I_{1}^{+}\right) . \cap C E\left(I_{1}^{-}\right) . \neq C E\left(I_{1}^{+} \cap I_{1}^{-}\right) . ;$for example, $I_{1}^{+} \wedge I_{1}^{-}$is a subspace in degree two of the left-hand side, but not of the right-hand side.

Diverging from [Beilinson 1980] we shall primarily use the following slightly different auxiliary construction (which we will later relate to the above one):

Definition 12. For $1 \leq p \leq n+1$ let

$$
{ }^{\otimes} T^{p}:=\coprod_{\substack{s_{1} \ldots s_{n} \in\{ \pm, 0\} \\ \operatorname{deg}\left(s_{1} \ldots s_{n}\right)=p}} C\left(I_{1}^{s_{1}} \cap I_{2}^{s_{2}} \cap \cdots \cap I_{n}^{s_{n}}\right) .
$$

and ${ }^{\otimes} T^{0}:=C(\mathfrak{g})$.

So, instead of the modified Chevalley-Eilenberg complex of Section 2 we just use the standard complexes for Lie homology with suitable coefficients. Clearly the morphism $I: C(\mathfrak{g})_{r} \rightarrow C(k)_{r+1}$ descends to morphisms

$$
\begin{aligned}
& C(\mathfrak{g})_{r} \supseteq C\left(I_{i}^{s_{i}}\right)_{r} \rightarrow C E\left(I_{i}^{s_{i}}\right)_{r+1} \subseteq C(k)_{r+1}, \\
& \underset{\in I_{i}^{s_{i}}}{f_{0} \otimes f_{1}} \wedge \cdots \wedge f_{r} \mapsto(-1)^{r} \underset{\in I_{i}^{s_{i}}}{f_{0}} \wedge f_{1} \wedge \cdots \wedge f_{r} .
\end{aligned}
$$

As we take intersections of Lie ideals on the left $C\left(I_{1}^{S_{1}} \cap \ldots\right)$, as in (3-2), the image lies in the intersection of the individual images, i.e., $C E\left(I_{1}^{s_{i}}\right)_{\bullet} \cap \cdots$, as in 
(3-1). As a result, we obtain morphisms

$$
{ }^{\otimes} T_{\bullet}^{p} \stackrel{I}{\rightarrow}{ }^{\wedge} T_{\bullet+1}^{p} \quad(\text { for all } p)
$$

and since they are a restriction of the map $I$ to subcomplexes, this is a morphism of complexes, and thus induces maps on homology.

\section{The cube complex}

Next, we shall define maps $\cdots \rightarrow{ }^{\otimes} T_{\bullet}^{2} \rightarrow{ }^{\otimes} T_{\bullet}^{1} \rightarrow^{\otimes} T_{\bullet}^{0} \rightarrow 0$, so that $\left({ }^{\otimes} T_{\bullet}\right)^{\bullet}$ becomes an exact superscript-indexed complex (of subscript-indexed complexes); and the same for ${ }^{\wedge} T_{\bullet}$. We begin by discussing ${ }^{\otimes} T_{\bullet}^{\bullet}$.

We define a $\mathfrak{g}$-module $N^{0}:=\mathfrak{g}$ and for $p \geq 1$

$$
N^{p}:=\coprod_{s_{1} \ldots s_{n} \in\{+,-, 0\}} I_{1}^{s_{1}} \cap I_{2}^{s_{2}} \cap \cdots \cap I_{n}^{s_{n}} \quad\left(\text { with } \operatorname{deg}\left(s_{1} \ldots s_{n}\right)=p\right) .
$$

We shall denote the components $f=\left(f_{s_{1} \ldots s_{n}}\right)$ of elements in $N^{p}$ with indices in terms of $s_{1}, \ldots, s_{n} \in\{+,-, 0\}$. Clearly $N^{p}=0$ for $p>n+1$. We shall treat all $N^{p}$ as $\mathfrak{g}$-modules and observe that

$$
{ }^{\otimes} T_{\bullet}^{p}=C\left(N^{p}\right)
$$

(by definition!), so by the functoriality and flatness ${ }^{1}$ of $C$. it suffices to construct an exact complex $N^{\bullet}$ out of the $N^{p}$ and then ${ }^{\otimes} T_{\bullet}^{p}$ will be an exact complex in $p$.

Example 13. For $n=1$ we have

$$
N^{2}=I_{1}^{0}, \quad N^{1}=I_{1}^{+} \oplus I_{1}^{-}
$$

and elements would be denoted $f=\left(f_{0}\right) \in N^{2}$ and $g=\left(g_{+}, g_{-}\right) \in N^{1}$. For $n=2$ we have

$$
\begin{aligned}
& N^{3}=I_{1}^{0} \cap I_{2}^{0}, \quad N^{2}=\left(\coprod_{s_{1} \in\{+,-\}} I_{1}^{s_{1}} \cap I_{2}^{0}\right) \oplus\left(\coprod_{s_{2} \in\{+,-\}} I_{1}^{0} \cap I_{2}^{s_{2}}\right) \\
& N^{1}=\coprod_{s_{1}, s_{2} \in\{+,-\}} I_{1}^{s_{1}} \cap I_{2}^{s_{2}} .
\end{aligned}
$$

We shall use the shorthand $s_{1} \ldots \pm \ldots s_{n}$ to indicate that in the $i$-th place we have $s_{i} \in\{+,-\}$, whatever $i$ may be at the moment. Similarly, $s_{1} \ldots 0 \ldots s_{n}$ will

\footnotetext{
${ }^{1}$ We just tensor $N^{p}$ with the vector spaces $\bigwedge^{i} \mathfrak{g}$. Being over a field, this preserves exact sequences.
} 
imply that $s_{i}=0$. Define $\mathfrak{g}$-module homomorphisms

$$
\begin{aligned}
\left(\partial_{i} f\right)_{s_{1} \ldots \pm \ldots s_{n}} & :=(-1)^{\#\left\{j \mid j>i \text { and } s_{j}=0\right\}} f_{s_{1} \ldots 0 \ldots s_{n}}, \\
\left(\partial_{i} f\right)_{s_{1} \ldots 0 \ldots s_{n}} & :=0 \\
\partial & :=\sum_{i=1}^{n} \partial_{i} .
\end{aligned}
$$

One checks easily that $\partial_{i}^{2}=0$ and $\partial_{i} \partial_{j}+\partial_{j} \partial_{i}=0$ for all $i, j=1, \ldots, n$. As a consequence, $\partial^{2}=0$. The components are given explicitly by

$$
(\partial f)_{s_{1} \ldots s_{n}}=\sum_{i=1}^{n}\left(\partial_{i} f\right)_{s_{1} \ldots s_{n}}=\sum_{\left\{i \mid s_{i}=+,-\right\}}(-1)^{\#\left\{j \mid j>i \text { and } s_{j}=0\right\}} f_{s_{1} \ldots 0 \ldots s_{n}} .
$$

Definition 14. Let $\left(A,\left(I_{i}^{ \pm}\right), \tau\right)$ be an $n$-fold cubically decomposed algebra over a field $k$. A system of good idempotents are pairwise commuting elements $P_{i}^{+} \in A$ for $i=1, \ldots, n$ such that for all $i$ :

(1) $P_{i}^{+2}=P_{i}^{+}$.

(2) $P_{i}^{+} A \subseteq I_{i}^{+}$.

(3) $P_{i}^{-} A \subseteq I_{i}^{-} \quad$ (where we define $P_{i}^{-}:=\mathbb{1}_{A}-P_{i}^{+}$).

We note that the $P_{i}^{-}$are also pairwise commuting idempotents and $P_{i}^{+}+P_{i}^{-}=\mathbb{1}_{A}$. Next, for $s_{i} \in\{+,-\}$ define $k$-vector space homomorphisms

$$
\begin{aligned}
\left(\varepsilon_{i} f\right)_{s_{1} \ldots s_{i} \ldots s_{n}}:=(-1)^{s_{i}} P_{i}^{s_{i}} \sum_{\gamma_{i} \in\{ \pm\}}(-1)^{\gamma_{i}} f_{s_{1} \ldots \gamma_{i} \ldots s_{n}}, \\
\left(\varepsilon_{i} f\right)_{s_{1} \ldots 0 \ldots s_{n}}:=0,
\end{aligned}
$$

where $(-1)^{ \pm}= \pm 1$. By direct calculation one verifies the identities $\varepsilon_{i}^{2}=\varepsilon_{i}$ and $\varepsilon_{i} \varepsilon_{j}=\varepsilon_{j} \varepsilon_{i}$ for all $i, j=1, \ldots, n$. Finally, define

$$
\begin{aligned}
& \left(H_{i} f\right)_{s_{1} \ldots 0 \ldots s_{n}}:=(-1)^{\#\left\{j \mid j>i \text { and } s_{j}=0\right\}} \sum_{\gamma_{i} \in\{ \pm\}} P_{i}^{-\gamma_{i}} f_{s_{1} \ldots \gamma_{i} \ldots s_{n}}, \\
& \left(H_{i} f\right)_{s_{1} \ldots \pm \ldots s_{n}}:=0 .
\end{aligned}
$$

The expression $P_{i}^{-\gamma_{i}}$ means $P_{i}^{-}$for $\gamma_{i}=+$ and $P_{i}^{+}$for $\gamma_{i}=-$. One checks that

$$
\begin{array}{rll}
H_{i}^{2}=0 & \text { and } & H_{i} H_{j}+H_{j} H_{i}=0, \\
\partial_{i} \varepsilon_{j}=\varepsilon_{j} \partial_{i} & \text { and } & H_{i} \varepsilon_{j}=\varepsilon_{j} H_{i}
\end{array}
$$

for all $i, j=1, \ldots, n$. Moreover, $\partial_{i} H_{j}+H_{j} \partial_{i}=0$ whenever $i \neq j$. In the special case $i=j$ one finds instead that

$$
\partial_{i} H_{i}+H_{i} \partial_{i}=\mathbb{1}-\varepsilon_{i} .
$$


Define $H:=H_{1}+\varepsilon_{1} H_{2}+\cdots+\varepsilon_{1} \varepsilon_{2} \cdots \varepsilon_{n-1} H_{n}$. Using the identities established above, one finds very easily

$$
H^{2}=0 \quad \text { and } \quad \partial H+H \partial=\mathbb{1}-\varepsilon_{1} \cdots \varepsilon_{n} .
$$

The fact $H^{2}=0$ was observed by the anonymous referee; it explains a certain cancellation in the proof of Proposition 24, which had been rather mysterious in an earlier version of this text.

Lemma 15. An explicit formula for $H$ is given by

$$
\begin{aligned}
(H f)_{s_{1} \ldots s_{n}}=(-1)^{\operatorname{deg}\left(s_{1} \ldots s_{n}\right)}(-1)^{s_{1}+\cdots+s_{b}} P_{1}^{s_{1}} \cdots P_{i}^{s_{b}} & \\
\times & \sum_{\gamma_{1} \ldots \gamma_{b+1} \in\{ \pm\}}(-1)^{\gamma_{1}+\cdots+\gamma_{b}} P_{b+1}^{-\gamma_{b+1}} f_{\gamma_{1} \ldots \gamma_{b+1} s_{b+2} \ldots s_{n}},
\end{aligned}
$$

where $b$ denotes the largest index such that $s_{1}, \ldots, s_{b} \in\{ \pm\}$ or $b=0$ if none (and so $s_{b+1}=0$ if $b<n ; b+1$ is the index of the leftmost zero).

Proof. One shows that

$\left(\varepsilon_{1} \cdots \varepsilon_{i} f\right)_{s_{1} \ldots s_{n}}=$

$$
\begin{cases}(-1)^{s_{1}+\cdots+s_{i}} P_{1}^{s_{1}} \cdots P_{i}^{s_{i}} & \\ \quad \times \sum_{\gamma_{1} \ldots \gamma_{i} \in\{ \pm\}}(-1)^{\gamma_{1}+\cdots+\gamma_{i}} f_{\gamma_{1} \ldots \gamma_{i} s_{i+1} \ldots s_{n}} & \text { for } s_{1}, \ldots, s_{i} \in\{ \pm\} \\ 0 & \text { if } 0 \in\left\{s_{1}, \ldots, s_{i}\right\}\end{cases}
$$

by evaluating $\left(\varepsilon_{j} \cdots \varepsilon_{i} f\right)$ inductively along $j=i, i-1, \ldots, 1$. Plug in $H_{i+1} f$ for $f$ to obtain

$$
\begin{aligned}
\left(\varepsilon_{1} \cdots \varepsilon_{i} H_{i+1} f\right)_{s_{1} \ldots s_{n}}=(-1)^{\#\left\{j \mid j>i+1 \text { and } s_{j}=0\right\}}(-1)^{s_{1}+\cdots+s_{i}} P_{1}^{s_{1}} \cdots P_{i}^{s_{i}} \\
\quad \times \sum_{\gamma_{1} \ldots \gamma_{i+1} \in\{ \pm\}}(-1)^{\gamma_{1}+\cdots+\gamma_{i}} P_{i+1}^{-\gamma_{i+1}} f_{\gamma_{1} \ldots \gamma_{i} \gamma_{i+1} s_{i+2} \ldots s_{n}}
\end{aligned}
$$

for $s_{1}, \ldots, s_{i} \in\{ \pm\}$ and $s_{i+1}=0$. Otherwise, (that is, for $0 \in\left\{s_{1}, \ldots, s_{i}\right\}$ or $\left.s_{i+1} \in\{ \pm\}\right)$, the respective component is zero. Thus,

$$
H_{s_{1} \ldots s_{n}}=\sum_{i=1}^{n}\left(\varepsilon_{1} \cdots \varepsilon_{i} H_{i+1} f\right)_{s_{1} \ldots s_{n}} .
$$

The summands with $i>b$ vanish since for them $0 \in\left\{s_{1}, \ldots, s_{i}\right\}$. The summands with $i<b$ vanish since for them $s_{i+1} \in\{ \pm\}$. Thus,

$$
H_{s_{1} \ldots s_{n}}=\left(\varepsilon_{1} \cdots \varepsilon_{b} H_{b+1} f\right)_{s_{1} \ldots s_{n}}
$$

and we use the above explicit formula. Note that $\#\left\{j \mid j>b+1\right.$ and $\left.s_{j}=0\right\}$ is just one below the total number of slots with value 0 since $s_{1}, \ldots, s_{b} \in\{ \pm\}$ and $s_{b+1}=0$. Thus, $(-1)^{\#\left\{j \mid j>i+1 \text { and } s_{j}=0\right\}}=(-1)^{\operatorname{deg}\left(s_{1} \ldots s_{n}\right)}$. 
The above maps are defined for $N^{p}$ in degrees $\geq 1$. We extend them to degree zero by defining the maps

$$
\hat{\partial}: N^{1} \rightarrow N^{0} \quad \text { and } \quad \hat{H}: N^{0} \rightarrow N^{1}
$$

as follows:

$$
\begin{aligned}
\hat{\partial} f & :=\sum_{s_{1} \ldots s_{n} \in\{+,-\}}(-1)^{s_{1}+\cdots+s_{n}} f_{s_{1} \ldots s_{n}}, \\
(\hat{H} f)_{s_{1} \ldots s_{n}} & :=(-1)^{s_{1}+\cdots+s_{n}} P_{1}^{s_{1}} \cdots P_{n}^{s_{n}} f .
\end{aligned}
$$

Along with these, we obtain the following crucial fact:

Lemma 16. Equipped with these morphisms,

$$
N^{\bullet}=\left[N^{n+1} \underset{H}{\stackrel{\partial}{\rightleftarrows}} N^{n} \underset{H}{\stackrel{\partial}{\rightleftarrows}} \cdots \underset{H}{\stackrel{\partial}{\rightleftarrows}} N^{1} \underset{\hat{H}}{\stackrel{\hat{\partial}}{\rightleftarrows}} N^{0}\right]_{n+1,0}
$$

is a complex of $\mathfrak{g}$-modules with differentials $\partial_{\bullet}($ resp. $\hat{\partial})$ and contracting homotopies $H_{.}($resp. $\hat{H})$ in the category of $k$-vector spaces.

Proof. The identities $\partial^{2}=0$ and $\hat{\partial} \circ \partial=0: N^{2} \rightarrow N^{0}$ are easy to check. Next, we confirm the contracting homotopy. We find $\partial H+H \partial=\mathbb{1}-\varepsilon_{1} \cdots \varepsilon_{n}$ by a telescope cancellation. For $f \in N^{i}$ with $i \geq 2$ for each component $f_{s_{1} \ldots s_{n}}$ there must be at least one $i$ with $s_{i}=0$ and thus $\left.\varepsilon_{1} \cdots \varepsilon_{n}\right|_{N^{i}}=0$ for $i \geq 2$. It remains to treat $i=0,1$. For $i=1$ we compute

$$
\hat{H} \hat{\partial} f=(-1)^{s_{1}+\cdots+s_{n}} P_{1}^{s_{1}} \cdots P_{n}^{s_{n}} \sum_{s_{1} \ldots s_{n} \in\{+,-\}}(-1)^{s_{1}+\cdots+s_{n}} f_{s_{1} \ldots s_{n}}=\varepsilon_{1} \cdots \varepsilon_{n} f
$$

(as in (4-6)). Thus, $\partial H+\hat{H} \hat{\partial}=\mathbb{1}$ on $N^{1}$. Finally, for $i=0$ we compute $\hat{\partial} \hat{H} f=f$.

Corollary 17. $0 \rightarrow{ }^{\otimes} T_{\bullet}^{n+1} \rightarrow{ }^{\otimes} T_{\bullet}^{n} \rightarrow \cdots \rightarrow{ }^{\otimes} T_{\bullet}^{0} \rightarrow 0$ with differential (and a contracting homotopy) induced by $\partial \otimes \mathrm{id}_{\wedge} \cdot \mathfrak{g}\left(\right.$ and $H \otimes \mathrm{id}_{\wedge} \cdot \mathfrak{g}$ ) is an exact complex (of complexes of $k$-vector spaces).

For the corollary, just use that tensoring with $\bigwedge^{r} \mathfrak{g}$ is exact.

\section{The cube complex, II}

Next, it would be nice to give a discussion of the ${ }^{\wedge} T_{\bullet}^{\bullet}$ parallel to the one for ${ }^{\otimes} T_{\bullet}^{\bullet}$ in the previous section. We can only do this to a limited extent, however.

Lemma 18. The definition

$$
(\partial f)_{s_{1} \ldots s_{n}}=\sum_{\left\{i \mid s_{i}=+,-\right\}}(-1)^{\#\left\{j \mid j>i \text { and } s_{j}=0\right\}} f_{s_{1} \ldots 0 \ldots s_{n}}
$$

turns ${ }^{\wedge} T_{0}$ : into a complex (of complexes of $k$-vector spaces) with respect to the superscript index. The morphisms $I:{ }^{\otimes} T_{\bullet}^{p} \rightarrow{ }^{\wedge} T_{\bullet+1}^{p}$ yield a morphism of complexes. 
Proof. Easy. Just check that the map $\partial$ is well-defined and satisfies $\partial^{2}=0$; in fact, exactly the same computation as in (4-2) applies. For the second claim, we just need to show that the map I commutes with the differential of either complex, but this is clear since the differentials are given by the same formula - compare (4-3) with (5-1).

The complex ${ }^{\wedge} T_{\bullet} \bullet$ is the central object in Beilinson's construction [1980]. We will use its analogue ${ }^{\otimes} T_{\bullet}$ as an auxiliary computational device. Firstly, let us explain Beilinson's construction. We need the following entirely homological tool:

Lemma 19. Suppose we are given an exact sequence

$$
S^{\bullet}=\left[S^{n+1} \rightarrow S^{n} \rightarrow \cdots \rightarrow S^{0}\right]_{n+1,0}
$$

with entries in $\mathbf{C h}^{+} \operatorname{Mod}_{k}$; that is, each $S^{i}=S^{i}$ is a bounded-below complex of k-vector spaces. ${ }^{2}$

(1) There is a second-quadrant homological spectral sequence $\left(E_{p, q}^{r}, d_{r}\right)$ converging to zero such that

$$
E_{p, q}^{1}=H_{q}\left(S_{\bullet}^{p}\right) \quad\left(d_{r}: E_{p, q}^{r} \rightarrow E_{p-r, q+r-1}^{r}\right) .
$$

(2) There is a first-quadrant cohomological spectral sequence $\left(E_{r}^{p, q}, d^{r}\right)$ converging to zero such that

$$
E_{1}^{p, q}=H^{q}\left(\operatorname{Hom}_{k}\left(S_{\bullet}^{p}, k\right)\right) \quad\left(d^{r}: E_{r}^{p, q} \rightarrow E_{r}^{p+r, q-r+1}\right) .
$$

(3) The following differentials are isomorphisms:

$$
d_{n+1}: E_{n+1,1}^{n+1} \rightarrow E_{0, n+1}^{n+1} \quad \text { and } \quad d^{n+1}: E_{n+1}^{0, n+1} \rightarrow E_{n+1}^{n+1,1} .
$$

(4) Suppose $H_{p}: S^{p} \rightarrow S^{p+1}$ is a contracting homotopy for $S^{\bullet}$. Then

$$
\left(d_{n+1}\right)^{-1}=H_{n} \delta_{1} H_{n-1} \cdots \delta_{n-1} H_{1} \delta_{n} H_{0}=H_{n} \prod_{i=1, \ldots, n}\left(\delta_{i} H_{n-i}\right)
$$

(where the last product depends on the ordering and refers to composition), and

$$
\left(d^{n+1}\right)^{-1}=H_{0}^{*} \delta_{n}^{*} H_{1}^{*} \cdots \delta_{1}^{*} H_{n}^{*}=H_{0}^{*} \prod_{i=n, \ldots, 1}\left(\delta_{i}^{*} H_{n+1-i}^{*}\right),
$$

where we write $f^{*}=\operatorname{Hom}_{k}(f, k)$ as a shorthand.

The construction is functorial in $S^{\bullet}$; that is, if $S^{\bullet} \rightarrow S^{\bullet}$ is a morphism of complexes as in our assumptions, then there are induced morphisms between their spectral sequences.

\footnotetext{
${ }^{2}$ One may alternatively view this as a bicomplex supported horizontally in degrees $[0, n+1]$, bounded from below, and whose rows are exact.
} 
Proof. Parts (1)-(3) are [Beilinson 1980, Lemma 1(a)]. More precisely, for (1) use the bicomplex spectral sequence for

$$
E_{p, q}^{0}=S_{q}^{p} \quad \text { and } \quad E_{0}^{p, q}=\operatorname{Hom}_{k}\left(S_{q}^{p}, k\right) .
$$

If we take differentials " $\rightarrow$ " for forming the $E^{0}$-page, the $E^{1}$-page vanishes since $S$. is exact (as a complex of complexes) and so the individual sequences of $k$-vector

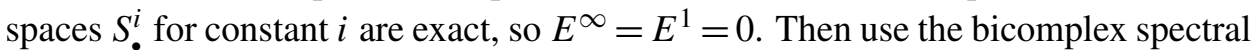
sequences with differential " $\downarrow$ " on the $E^{0}$-page for our claim. It also converges to zero then; (2) is analogous. For (3), the bicomplex is horizontally supported in $[0, n+1]$. For (4), diagram chase.

We combine Lemma 18 with Lemma 19: Apply the latter to $S_{q}^{p}:={ }^{\wedge} T_{q}^{p}$; we denote the resulting spectral sequence by ${ }^{\wedge} E_{\bullet, \bullet}$. The fact that the (bi)complex of Lemma 19 is supported horizontally in $[n+1,0]$ homologically (i.e., for ${ }^{\wedge} E_{\bullet, \bullet}^{\bullet}$ ) and in $[0, n+1]$ cohomologically (i.e., for ${ }^{\wedge} E_{\bullet}^{\bullet \bullet \bullet}$ ) implies that we have edge morphisms

$$
\begin{gathered}
\rho_{1}:{ }^{\wedge} E_{n+1,1}^{n+1} \rightarrow{ }^{\wedge} E_{n+1,1}^{1} \text { and } \rho_{2}:{ }^{\wedge} E_{0, n+1}^{1} \rightarrow{ }^{\wedge} E_{0, n+1}^{n+1}, \\
\wp_{1}:{ }^{\wedge} E_{n+1}^{0, n+1} \rightarrow{ }^{\wedge} E_{1}^{0, n+1} \text { and } \wp_{2}:{ }^{\wedge} E_{1}^{n+1,1} \rightarrow{ }^{\wedge} E_{n+1}^{n+1,1} .
\end{gathered}
$$

Next, we identify the objects involved: Using Lemma 9 we compute

$$
\begin{aligned}
& { }^{\wedge} E_{0, n+1}^{1}=H_{n+1}\left({ }^{\wedge} T_{\bullet}^{0}\right)=H_{n+1}(C E(\mathfrak{g}) .) \cong H_{n+1}(\mathfrak{g}, k), \\
& { }^{\wedge} E_{n+1,1}^{1}=H_{1}\left({ }^{\wedge} T_{\bullet}^{n+1}\right)=H_{1}\left(\bigcap_{i=1, \ldots, n} \bigcap_{s_{i} \in\{ \pm\}} C E\left(I_{i}^{s_{i}}\right) \cdot\right)=I_{\mathrm{tr}} /\left[I_{\mathrm{tr}}, \mathfrak{g}\right], \\
& { }^{\wedge} E_{1}^{n+1,1}=\operatorname{Hom}_{k}\left(I_{\mathrm{tr}} /\left[I_{\mathrm{tr}}, \mathfrak{g}\right], k\right) \text { and }{ }^{\wedge} E_{1}^{0, n+1}=H^{n+1}(\mathfrak{g}, k) .
\end{aligned}
$$

Definition 20 [Beilinson 1980]. Let $\left(A,\left(I_{i}^{ \pm}\right), \tau\right)$ be an $n$-fold cubically decomposed algebra over a field $k$ and $\mathfrak{g}:=A_{\text {Lie }}$ its Lie algebra. Define

$$
\operatorname{res}_{*}: H_{n+1}(\mathfrak{g}, k) \rightarrow k \quad \operatorname{res}_{*}:=\tau \circ \rho_{1} \circ\left(d_{n+1}\right)^{-1} \circ \rho_{2}
$$

and

$$
\text { res* }^{*} k \rightarrow H^{n+1}(\mathfrak{g}, k) \quad \operatorname{res}^{*}(1):=\left(\wp_{1} \circ\left(d^{n+1}\right)^{-1} \circ \wp_{2}\right) \tau,
$$

where for res* we read $\tau$ as an element of $E_{1}^{n+1,1}$. We will call $\phi:=\operatorname{res}^{*}(1)$ the Tate extension class.

In the case $n=1$ it would also be justified to name this cohomology class after [Kac and Peterson 1981]; it also appears in the works of the Japanese school, e.g., [Jimbo and Miwa 1983].

Remark 21. It follows from the construction of res $_{*}$ and res* that

$$
\operatorname{res}^{*}(\alpha)\left(X_{0} \wedge \cdots \wedge X_{n}\right)=\alpha \operatorname{res}_{*} X_{0} \wedge \cdots \wedge X_{n} .
$$


Now we would like to compute these maps explicitly. Clearly, the most elusive map in the construction is the differential $d_{n+1}$ (resp. $\left.d^{n+1}\right)$. We can render it explicit using Lemma 19(4) as soon as we have an explicit contracting homotopy available. However, it seems to be quite difficult to construct such a homotopy for the complex ${ }^{\wedge} T^{\bullet}$. On the other hand, we $d o$ have such a contracting homotopy for ${ }^{\otimes} T^{\bullet}$ by Lemma 16 and its corollary. Luckily for us, these complexes are closely connected. We may apply Lemma 19 also to $S_{q}^{p}:={ }^{\otimes} T_{q-1}^{p}$; this time denote the resulting spectral sequence by ${ }^{\otimes} E_{\bullet, \bullet}$. We easily compute

$$
\begin{aligned}
& { }^{\otimes} E_{0, n+1}^{1}=H_{n+1}\left({ }^{\otimes} T_{\cdot-1}^{0}\right)=H_{n}\left(C(\mathfrak{g})_{\bullet}\right) \cong H_{n}(\mathfrak{g}, \mathfrak{g}), \\
& { }^{\otimes} E_{n+1,1}^{1}=H_{1}\left({ }^{\otimes} T_{\bullet-1}^{n+1}\right)=H_{0}\left(C\left(\bigcap_{i=1, \ldots, n} \bigcap_{s_{i} \in\{ \pm\}} I_{i}^{s_{i}}\right)\right)=I_{\mathrm{tr}} /\left[I_{\mathrm{tr}}, \mathfrak{g}\right], \\
& { }^{\otimes} E_{1}^{n+1,1}=\operatorname{Hom}_{k}\left(I_{\mathrm{tr}} /\left[I_{\mathrm{tr}}, \mathfrak{g}\right], k\right) \text { and }{ }^{\otimes} E_{1}^{0, n+1}=H^{n}\left(\mathfrak{g}, \mathfrak{g}^{*}\right) .
\end{aligned}
$$

We note that some groups even agree with $\operatorname{their}^{\wedge} T_{q}^{p}$-counterpart, as we had already observed in (2-4).

Definition 22. Write ${ }^{\otimes}$ res $_{*}: H_{n}(\mathfrak{g}, \mathfrak{g}) \rightarrow k$ and ${ }^{\otimes}$ res* $^{*}(1) \in H^{n}\left(\mathfrak{g}, \mathfrak{g}^{*}\right)$ for the counterparts of res $_{*}$, res* in Definition 20 using ${ }^{\otimes} E$ instead of ${ }^{\wedge} E$.

Lemma 23 (Compatibility). The morphism of bicomplexes ${ }^{\otimes} T_{\bullet}^{\bullet} \stackrel{I}{\rightarrow}^{\wedge} T_{\bullet+1}^{\bullet}$ induces a commutative diagram

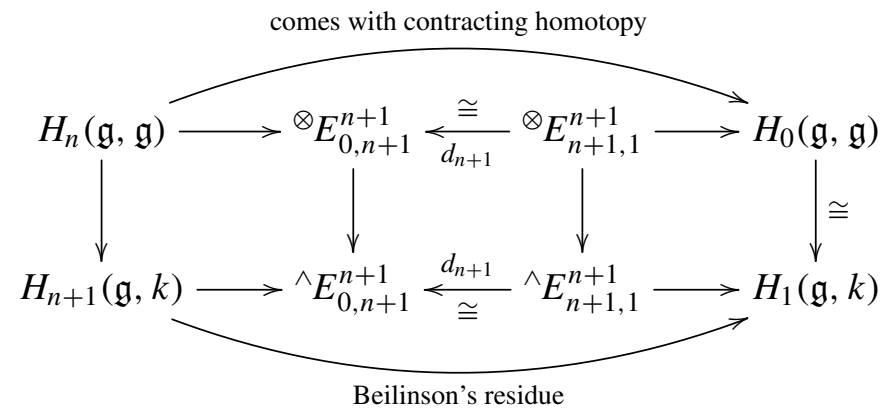

Proof. We had already observed in Lemma 18 that the morphisms $I$ induce a morphism of bicomplexes. The spectral sequences ${ }^{\otimes} E_{\mathbf{0}, \bullet}$ and ${ }^{\wedge} E_{\bullet, \bullet}^{\bullet}$ both arise from Lemma 19, so by the functoriality of the construction we get an induced morphism of spectral sequences. In particular, all squares

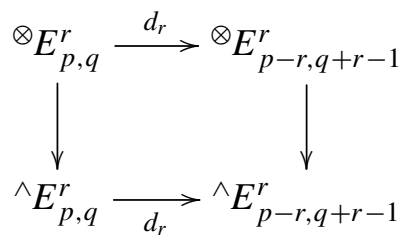


commute, giving the middle square in our claim. The same applies to the edge maps, giving the outer squares.

Absolutely analogously we obtain a cohomological counterpart

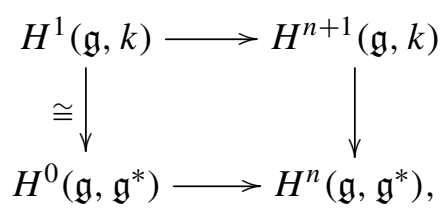

where we have a contracting homotopy for the lower row. We leave the details of this formulation to the reader.

\section{Concrete formalism}

Let $\left(A,\left(I_{i}^{ \pm}\right), \tau\right)$ be an $n$-fold cubically decomposed algebra over a field $k$. In Section 5 we have constructed a canonical morphism

$$
\begin{aligned}
& \operatorname{res}_{*}: H_{n+1}(\mathfrak{g}, k) \rightarrow k \\
& \begin{array}{c}
\uparrow \\
H_{n}(\mathfrak{g}, \mathfrak{g}),
\end{array}
\end{aligned}
$$

where $\mathfrak{g}:=A_{\text {Lie }}$ is the Lie algebra associated to $A$. By Lemma 23 , its values on the image of $H_{n}(\mathfrak{g}, \mathfrak{g}) \rightarrow H_{n+1}(\mathfrak{g}, k)$ can be computed via ${ }^{\otimes}$ res $_{*}$. In this section we will obtain an explicit formula for the latter morphism.

Given the definition of ${ }^{\otimes}$ res $_{*}$, Lemma 19(4) tells us that it can be given explicitly in terms of differentials of the ordinary Chevalley-Eilenberg complexes $C(-)$. (see Section 2) and contracting homotopies of the cube complex $N^{\bullet}$ (see Lemma 16 and its corollary), namely

$$
{ }^{\otimes} \mathrm{res}_{*}=\tau \circ \rho_{1} \circ\left({ }^{\otimes} d_{n+1}\right)^{-1} \circ \rho_{2}=\tau \circ \rho_{1} H \prod_{i=1, \ldots, n}\left(\delta_{i} H\right) \rho_{2}
$$

via the spectral sequence ${ }^{\otimes} E_{., .}$. The contracting homotopy $H$ depends on the choice of a good system of idempotents; see Definition 14. Different choices will yield formulas that may look different, but as ${ }^{\otimes}$ res $_{*}$ (just like res ${ }_{*}$ itself) was defined entirely independently of the choice of any idempotents, all such formulas actually must agree.

Suppose a representative $\theta:=f_{0} \otimes f_{1} \wedge \cdots \wedge f_{n}$ with $f_{0}, \ldots, f_{n} \in N^{0}$ is given (note that $N^{0}$ equals $\mathfrak{g}$ as a left- $U \mathfrak{g}$-module by definition, so it is valid to treat all $f_{i}$ on equal footing). We shall compute ${ }^{\otimes} \operatorname{res}_{*} \theta$ in several steps, starting with $\theta_{0, n}:=\rho_{2} \theta$, then following 


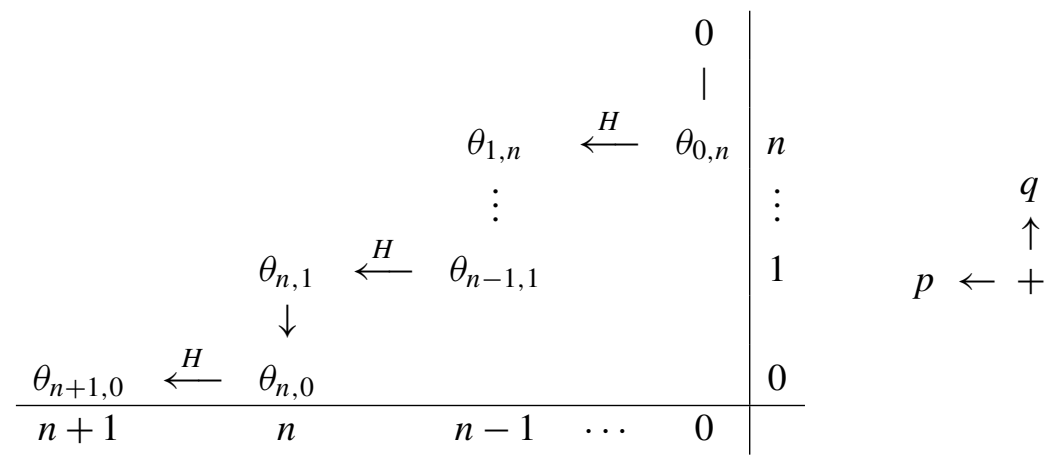

as prescribed by (6-1). This graphical arrangement elucidates the position of the term of each step in the computation in the spectral sequence from which (6-1) originates - see Lemma 19. However, for us each $\theta_{*, *}$ will be an $E^{0}$-page representative of the respective $E^{*}$-page term. Finally ${ }^{\otimes} \operatorname{res}_{*} \theta=\tau \rho_{1} \theta_{n+1,0}$. We note that $\rho_{1}, \rho_{2}$ are just edge maps, that is, an inclusion of a subobject and a quotient surjection. Hence, as we work with explicit representatives anyway, the operation of these maps is essentially invisible (e.g., in the quotient case it just means that our representative generates a larger equivalence class).

We will need a convenient notation for elements of this complex.

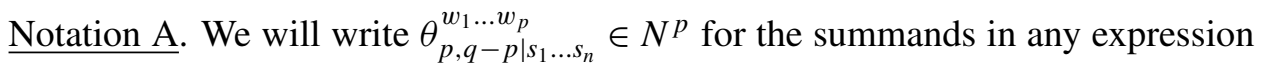
of the shape

$$
\theta_{p, q-p}=\sum_{\substack{w_{1} \ldots w_{p} \\ \in\{1, \ldots, n\}}} \sum_{s_{1} \ldots s_{n}} \theta_{p, q-p \mid s_{1} \ldots s_{n}}^{w_{1} \ldots w_{p}} \otimes f_{1} \wedge \cdots \wedge \widehat{f}_{w_{1}} \wedge \cdots \wedge{\widehat{f_{w}}}_{w_{p}} \wedge \cdots \wedge f_{n}
$$

where

- $(p, q-p)$ denotes the location of the element in the bicomplex as in (6-2),

- $s_{1}, \ldots, s_{n} \in\{0,+,-\}$ denotes the component (= direct summand) of $N^{p}$ as in (4-1), $f_{1}, \ldots, f_{n} \in \mathfrak{g}$,

- the additional superscripts $w_{1}, \ldots, w_{p} \in\{1, \ldots, n\}$ are used to indicate the omission of wedge factors.

Note that the values $\theta_{p, q \mid s_{1} \ldots s_{n}}^{w_{1} \ldots w_{p}}$ are not necessarily uniquely determined since the individual wedge tails need not be linearly independent.

Notation B. We also need a shorthand for the summands in any expression of the shape

$$
\begin{aligned}
\theta_{p, q-p-1}= & \sum_{\substack{w_{1} \ldots w_{p}, w_{a}, w_{b} \\
\in\{1, \ldots, n\}}} \sum_{s_{1} \ldots s_{n}} \theta_{p, q \mid s_{1} \ldots s_{n}}^{w_{1} \ldots w_{p} \| w_{a}, w_{b}} \\
& \otimes\left[f_{w_{a}}, f_{w_{b}}\right] \wedge f_{1} \wedge \cdots \widehat{f}_{w_{1}} \cdots \widehat{f}_{w_{a}} \cdots \widehat{f}_{w_{b}} \cdots \widehat{f}_{w_{p}} \cdots \wedge f_{n} .
\end{aligned}
$$


Again $s_{1}, \ldots, s_{n}$ denotes the component in $N^{p}, w_{1}, \ldots, w_{p}$ omitted wedge factors. Moreover, $w_{a}$ and $w_{b}$ denote two additional omitted wedge factors and simultaneously indicate that $\left[f_{w_{a}}, f_{w_{b}}\right]$ appears as an additional wedge factor. As for the previous notation, the elements $\theta_{p, q \mid s_{1} \ldots s_{n}}^{w_{1} \ldots w_{p} \| w_{a}, w_{b}} \in N^{p}$ are not uniquely determined. We will explain how these expressions arise soon.

Combinatorial preparation: We define for arbitrary $1 \leq p \leq n$ and $w_{1}, \ldots, w_{p} \in$ $\{1, \ldots, n\}$ a sign function (generalizing the sign of a permutation):

$$
\rho\left(w_{1}, \ldots, w_{p}\right):=(-1)^{\sum_{k=1}^{p} \sum_{j<k} \delta_{w_{j}<w_{k}}} .
$$

By abuse of language we do not carry the value $p$ in the notation for $\rho$ as it will always be clear from the number of arguments which variant is used. It is easy to

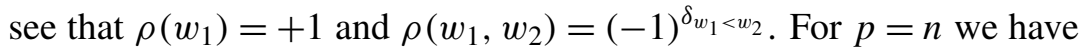

$$
\rho\left(w_{1}, \ldots, w_{n}\right)=\operatorname{sgn}\left(\begin{array}{ccc}
1 & \cdots & n \\
w_{1} & \cdots & w_{n}
\end{array}\right) .
$$

We shall need the inductive formula (which is easy to check by induction)

$$
(-1)^{\#\left\{w_{i} \mid 1 \leq i \leq p \text { s.t. } w_{i}<w_{p+1}\right\}} \rho\left(w_{1}, \ldots, w_{p}\right)=\rho\left(w_{1}, \ldots, w_{p+1}\right) .
$$

Proposition 24. Suppose $\theta:=f_{0} \otimes f_{1} \wedge \cdots \wedge f_{n}$ with $f_{i} \in N_{0}=\mathfrak{g}$. Moreover, suppose $P_{1}^{+}, \ldots, P_{n}^{+}$is a good system of idempotents as in Definition 14. Then for every $p \geq 0$ the element $\theta_{p+1, q}$ is of the shape as in (6-3) and for $\gamma_{1} \ldots \gamma_{n-p} \in\{+,-\}$ we have

$$
\begin{aligned}
& \theta_{p+1, q \mid \gamma_{1} \ldots \gamma_{n-p}}^{w_{1} \ldots w_{p}} \underbrace{0 \ldots \ldots 0}_{p} \\
& =(-1)^{\sum_{u=1}^{p-1}(u+1)}(-1)^{w_{1}+\cdots+w_{p}} \rho\left(w_{1}, \ldots, w_{p}\right)(-1)^{\gamma_{1}+\cdots+\gamma_{n-p}} P_{1}^{\gamma_{1}} \cdots P_{n-p}^{\gamma_{n-p}} \times \\
& \quad \sum_{\gamma_{n-p+1}^{*} \cdots \gamma_{n}^{*} \in\{ \pm\}}(-1)^{\gamma_{n-p+1}^{*}+\cdots+\gamma_{n}^{*}}\left(P_{n-p+1}^{\left(-\gamma_{n-p+1}^{*}\right)} \operatorname{ad}\left(f_{w_{p}}\right) P_{n-p+1}^{\gamma_{n-p+1}^{*}}\right) \cdots\left(P_{n}^{\left(-\gamma_{n}^{*}\right)} \operatorname{ad}\left(f_{w_{1}}\right) P_{n}^{\gamma_{n}^{*}}\right) f_{0} .
\end{aligned}
$$

Here $\rho\left(w_{1}, \ldots, w_{p}\right)$ is the sign function defined in (6-5). For $p=0$ the expression $\rho\left(w_{1}, \ldots, w_{p}\right)$ and the whole sum $\left(\sum_{\{ \pm\}}(\cdots)\right)$ in $\left(\sum_{\{ \pm\}}(\cdots)\right) f_{0}$ should be read as +1 (giving the right-hand side of (6-8) below).

- Note that no terms of the shape as in (6-4) appear. This is not entirely obvious in view of the definition of $\delta^{[2]}-\operatorname{see}(2-1)$.

- The formula does not compute $\theta_{p+1, q \mid s_{1} \ldots s_{n}}^{w_{1} \ldots w_{p}}$ for arbitrary $s_{1} \ldots s_{n}$ of degree $p+1$. This is due to the fact that we only have further use for the ones treated.

- For $p \leq 1 \mathrm{read} \sum_{u=1}^{p-1}(u+1)$ as zero. 
Proof. We prove this by induction. For $p=0$ the claim reads

$$
\theta_{1, q \mid \gamma_{1} \ldots \gamma_{n}}=(-1)^{\gamma_{1}+\cdots+\gamma_{n}} P_{1}^{\gamma_{1}} \cdots P_{n}^{\gamma_{n}} f_{0}
$$

and in view of (4-7) this proves the claim in this case. Now we proceed by induction. Assume the case $p$ is settled, that is, in the notation of (6-3),

$$
\theta_{p+1, q \mid \gamma_{1} \ldots \gamma_{n-p}}^{w_{1} \ldots w_{p}} \underbrace{0 \ldots 0}_{p}
$$

is exactly as in our claim. Next, we need to apply the differential $\delta_{q}=\delta_{q}^{[1]}+\delta_{q}^{[2]}$ of the Chevalley-Eilenberg resolution - see (2-1). The contribution of $\delta_{q}^{[1]}$ will be relevant, but for $\delta_{q}^{[2]}$ we shall see that (after applying the next contracting homotopy) the contribution vanishes. We treat each $\delta^{[i]}, i=1,2$ separately:

(1) Consider $\delta_{q}^{[1]}$ in (2-1). The sum $\Sigma_{i}$ loc. cit. maps components indexed by $w_{1}, \ldots, w_{p}$ to components of $\delta^{[1]} \theta_{p, q}$, indexed by $w_{1}, \ldots, w_{p}$ and an additional $w_{p+1} \in\{1, \ldots, n\} \backslash\left\{w_{1}, \ldots, w_{p}\right\}$ - they correspond to the summands of $\delta^{[1]} \theta_{p, q}$ and to the additional omitted wedge factor, respectively. Moreover, the formula imposes signs $(-1)^{i+1}$, but here $i$ depends on the numbering of the wedges $(\cdots \wedge \cdots \wedge \cdots)$. In the notation of (6-3) the subscript $j$ of $f_{j}$ does not necessarily indicate the $f_{j}$ sits in the $j$-th wedge, due to the possible omission of wedge factors $f_{w_{1}}, \ldots, f_{w_{p}}$ on the left-hand side of it. To compensate for that in the following computation the term $(-1)^{\#\left\{w_{i} \mid 1 \leq i \leq p \text { s.t. } w_{i}<w_{p+1}\right\}}$ appears, sign-counting the omission on the left of the new-to-be-omitted $w_{p+1}$ in the component of $\delta^{[1]} \theta_{p+1, q}$. As $p$ remains constant, the indexing $\gamma_{1} \ldots \gamma_{n-p} 0 \ldots 0$ remains unaffected. We get the expression

$$
\begin{aligned}
& \left(\delta^{[1]} \theta_{p+1, q}\right)_{p+1, q-1 \mid \gamma_{1} \ldots \gamma_{n-p}}^{w_{1} \ldots w_{p} w_{p+1}} \underbrace{0 \ldots 0}_{p} \\
& =(-1)^{\sum_{u=1}^{p-1}(u+1)}(-1)^{w_{p+1}+1}(-1)^{\#\left\{w_{i} \mid 1 \leq i \leq p \text { s.t. } w_{i}<w_{p+1}\right\}} \operatorname{ad}\left(f_{w_{p+1}}\right) \\
& \times(-1)^{w_{1}+\cdots+w_{p}} \rho\left(w_{1}, \ldots, w_{p}\right)(-1)^{\gamma_{1}+\cdots+\gamma_{n-p}} P_{1}^{\gamma_{1}} \cdots P_{n-p}^{\gamma_{n-p}} \\
& \quad \times \sum_{\gamma_{n-p+1}^{*} \cdots \gamma_{n}^{*} \in\{ \pm\}}(-1)^{\gamma_{n-p+1}^{*}+\cdots+\gamma_{n}^{*}}\left(P_{n-p+1}^{\left(-\gamma_{n-p+1}^{*}\right)} \text { ad } f_{w_{p}} P_{n-p+1}^{\gamma_{n-p+1}^{*}}\right) \cdots\left(P_{n}^{\left(-\gamma_{n}^{*}\right)} \text { ad } f_{w_{1}} P_{n}^{\gamma_{n}^{*}}\right) f_{0} .
\end{aligned}
$$

Next, we need to apply the contracting homotopy $H: N^{p+1} \rightarrow N^{p+2}$. We have $p+1 \geq 1$, so (4-5) applies. Note that for an index $\gamma_{1}^{\dagger} \ldots \gamma_{n-p-1}^{\dagger} 0 \ldots 0$ with $\gamma_{1}^{\dagger} \ldots \gamma_{n-p-1}^{\dagger} \in\{ \pm\}$ and $p+1$ zeros (i.e., an index of degree $p+2$; compare (4-1)), the corresponding index with one fewer 0 has degree $p+1$. Indices of the latter type have been dealt with above. We obtain 


$$
\begin{aligned}
& \left(H \delta^{[1]} \theta_{p+1, q}\right) \begin{array}{l}
w_{1} \ldots w_{p} w_{p+1} \\
p+2, q-1 \mid \gamma_{1}^{\dagger} \ldots \gamma_{n-p-1}^{\dagger} \underbrace{0 \ldots 0}_{p+1}
\end{array} \\
& =(-1)^{p}(-1)^{\gamma_{1}^{\dagger}+\cdots+\gamma_{n-p-1}^{\dagger}} P_{1}^{\gamma_{1}^{\dagger} \ldots P_{n-p-1}^{\gamma_{n-p-1}^{\dagger}}} \\
& \quad \times \sum_{\gamma_{1}, \ldots, \gamma_{(n-p-1)+1} \in\{ \pm\}}(-1)^{\gamma_{1}+\cdots+\gamma_{n-p-1}} P_{(n-p-1)+1}^{-\gamma_{(n-p-1)+1}}\left(\delta \theta_{p+1, q}\right)_{p+1, q-1 \mid \gamma_{1} \ldots \gamma_{n-p} \underbrace{0 \ldots 0}_{p}}^{w_{1} \ldots w_{p+1}}
\end{aligned} .
$$

In principle the first factor is $(-1)^{\operatorname{deg}(\cdots)}=(-1)^{p+2}$, but switching to $p$ preserves the correct sign. Next, we expand this using our previous computation and obtain (by noting that many signs are squares and thus +1 )

$$
\begin{aligned}
& =(-1)^{\sum_{u=1}^{p-1}(u+1)}(-1)^{p+1}(-1)^{\gamma_{1}^{\dagger}+\cdots+\gamma_{n-p-1}^{\dagger}}(-1)^{\#\left\{w_{i} \mid 1 \leq i \leq p \text { s.t. } w_{i}<w_{p+1}\right\}} \\
& \times(-1)^{w_{1}+\cdots+w_{p+1}} \rho\left(w_{1}, \ldots, w_{p}\right) P_{1}^{\gamma_{1}^{\dagger}} \ldots P_{n-p-1}^{\gamma_{n-p-1}^{\dagger}} \\
& \times \sum_{\gamma_{n-p} \in\{ \pm\}}(-1)^{\gamma_{n-p}}\left(\sum_{\gamma_{1} \ldots \gamma_{n-p-1} \in\{ \pm\}} P_{1}^{\gamma_{1}} \cdots P_{n-p-1}^{\gamma_{n-p-1}}\right) P_{n-p}^{-\gamma_{n-p}} \operatorname{ad}\left(f_{w_{p+1}}\right) P_{n-p}^{\gamma_{n-p}} \\
& \times \sum(-1)^{\gamma_{n-p+1}^{*}+\cdots+\gamma_{n}^{*}} \\
& \gamma_{n-p+1}^{*} \cdots \gamma_{n}^{*} \in\{ \pm\} \quad \times\left(P_{n-p+1}^{\left(-\gamma_{n-p+1}^{*}\right)} \operatorname{ad}\left(f_{w_{p}}\right) P_{n-p+1}^{\gamma_{n-p+1}^{*}}\right) \cdots\left(P_{n}^{\left(-\gamma_{n}^{*}\right)} \operatorname{ad}\left(f_{w_{1}}\right) P_{n}^{\gamma_{n}^{*}}\right) f_{0} .
\end{aligned}
$$

The sum in parentheses is the identity since for all $i$ we have $P_{i}^{+}+P_{i}^{-}=\mathbb{1}$ by Definition 14. Up to the naming of the indices, and after using (6-7), this is exactly our claim in the case $p+1$ (and this is true despite the fact that we have only considered $\delta^{[1]}$ so far, because we shall next show that the contribution from $H \circ \delta^{[2]}$ vanishes).

(2) Consider $\delta_{q}^{[2]}$ in (2-1). Using the notation of (6-3) we may write

$$
\theta_{p+1, q}=\bigoplus_{\operatorname{deg}\left(s_{1} \ldots s_{n}\right)=p+1} \sum_{\begin{array}{c}
w_{1} \ldots w_{p} \\
\in\{1, \ldots, n\}, \\
\text { pairw. diff. }
\end{array}} \theta_{p+1, q \mid s_{1} \ldots s_{n}}^{w_{1} \ldots w_{p}} \otimes f_{1} \wedge \widehat{f}_{w_{1}} \cdots \widehat{f}_{w_{p}} \wedge f_{n}
$$

Therefore

$$
\begin{aligned}
& \delta^{[2]} \theta_{p+1, q} \bigoplus_{\operatorname{deg}\left(s_{1} \ldots s_{n}\right)=p+1} \sum_{\substack{\underbrace{}_{w_{1} \ldots w_{p}} \in 1, \ldots, n\}, \in\{1, \ldots, n\} \backslash\left\{w_{1} \ldots w_{p}\right\} \\
\text { pairw. diff. }}} \sum_{\substack{w_{p+1}<w_{p+2}\\
}}(-1)^{w_{p+1}+w_{p+2}} \\
& \quad \times(-1)^{\#\left\{w_{i} \mid 1 \leq i \leq p \text { s.t. } w_{i}<w_{p+1}\right\}}(-1)^{\#\left\{w_{i} \mid 1 \leq i \leq p \text { s.t. } w_{i}<w_{p+2}\right\}} \\
& \quad \times \theta_{p+1, q \mid s_{1} \ldots s_{n}}^{w_{1} \ldots w_{p}} \otimes\left[f_{w_{p+1}}, f_{w_{p+2}}\right] \wedge f_{1} \wedge \widehat{f}_{w_{1}} \cdots \widehat{f}_{w_{p+1}} \cdots \widehat{f}_{w_{p+2}} \cdots \widehat{f}_{w_{p}} \wedge f_{n} .
\end{aligned}
$$


The two powers of -1 on the middle line of the right-hand side appear since the original summand in $\delta^{[2]}$ carries the sign $(-1)^{i+j}$, so we need to compute the number of the wedge slot correctly, respecting the omitted wedge factors; compare with the discussion in the first part of this proof. We observe that the first wedge factor remains unchanged under $\delta^{[2]}$. Hence, when we apply the contracting homotopy $H$ in this induction step and in the next again, the summand will vanish thanks to $H^{2}=0$; see (4-4). It will not do harm to verify this explicitly: We use the notation of (6-4) and write the above in terms of

$$
\begin{aligned}
& \left(\delta^{[2]} \theta_{p+1, q}\right)_{p+1, q-1 \mid s_{1} \ldots s_{n}}^{w_{1} \ldots w_{p} \| w_{p+1}, w_{p+2}} \\
& =(-1)^{w_{p+1}+w_{p+2}}(-1)^{\#\left\{w_{i} \mid 1 \leq i \leq p \text { s.t. } w_{i}<w_{p+1}\right\}}(-1)^{\#\left\{w_{i} \mid 1 \leq i \leq p \text { s.t. } w_{i}<w_{p+2}\right\}} \theta_{p+1, q \mid s_{1} \ldots s_{n}}^{w_{1} \ldots w_{p}} .
\end{aligned}
$$

Next, we apply the map $H: N^{p+1} \rightarrow N^{p+2}$ of (4-5). Then for indices $s_{1} \ldots s_{n}=$ $\gamma_{1}^{\dagger} \ldots \gamma_{n-p-1}^{\dagger} 0 \ldots 0$ and $\gamma_{1}^{\dagger} \ldots \gamma_{n-p-1}^{\dagger} \in\{ \pm\}$ (which is of degree $p+2$ ) we obtain the expression

$$
\begin{gathered}
\left(H \delta^{[2]} \theta_{p+1, q}\right)_{p+2, q-1 \mid \gamma_{1}^{\dagger} \ldots \gamma_{n-p-1}^{\dagger} \underbrace{w_{1} \ldots w_{p} \| w_{p+1}, w_{p+2}}_{p+1}}^{0 \ldots 0} \\
=P_{1}^{\gamma_{1}^{\dagger}} \cdots P_{n-p-1}^{\gamma_{n-p-1}^{\dagger}} \sum_{\gamma_{1} \ldots \gamma_{n-p} \in\{ \pm\}}(-1)^{(\cdots)} P_{n-p}^{-\gamma_{n-p}} \theta_{p+1, q \mid \gamma_{1} \ldots \gamma_{n-p}}^{w_{1} \ldots w_{p}} \underbrace{0 \ldots 0}_{p},
\end{gathered}
$$

where we have plugged in our previous computation and started to disregard the precise sign. We know the last term of this expression by our induction hypothesis and therefore obtain

$$
\begin{aligned}
=P_{1}^{\gamma_{1}^{\dagger}} \cdots P_{n-p-1}^{\gamma_{n-p-1}^{\dagger}} \sum_{\gamma_{1} \ldots \gamma_{n-p} \in\{ \pm\}} \sum_{\gamma_{n-p+1}^{*} \cdots \gamma_{n}^{*} \in\{ \pm\}}(-1)^{(\ldots)} \frac{P_{n-p}^{-\gamma_{n-p}} P_{1}^{\gamma_{1}} \cdots P_{n-p}^{\gamma_{n-p}}}{} \\
\quad \times\left(P_{n-p+1}^{\left(-\gamma_{n-p+1}^{*}\right)} \operatorname{ad}\left(f_{w_{p}}\right) P_{n-p+1}^{\gamma_{n-p+1}^{*}}\right) \cdots\left(P_{n}^{\left(-\gamma_{n}^{*}\right)} \operatorname{ad}\left(f_{w_{1}}\right) P_{n}^{\gamma_{n}^{*}}\right) f_{0} .
\end{aligned}
$$

As the $P_{1}^{+}, \ldots, P_{n}^{+}$commute pairwise, the same holds for all $P_{1}^{ \pm}, \ldots, P_{n}^{ \pm}$(by Definition 14). Thus, the underlined expression can be rearranged to

$$
P_{n-p}^{-\gamma_{n-p}} P_{n-p}^{\gamma_{n-p}} \cdots
$$

But

$$
P_{i}^{+} P_{i}^{-}=P_{i}^{+}\left(\mathbb{1}-P_{i}^{+}\right)=0
$$

because $P_{i}^{+}$is an idempotent. The same holds for $P_{i}^{-} P_{i}^{+}$. Hence, in all the indices $s_{1}, \ldots, s_{n}$ relevant for our claim $H \delta^{[2]} \theta_{p+1, q}$ is zero.

This readily implies the following key computation: 
Theorem 25 (main theorem). Let $\left(A,\left(I_{i}^{ \pm}\right), \tau\right)$ be an $n$-fold cubically decomposed algebra over a field $k$. Then

$$
\begin{aligned}
& { }^{\otimes} \operatorname{res}_{*}\left(f_{0} \otimes f_{1} \wedge \cdots \wedge f_{n}\right) \\
& =-(-1)^{\frac{(n-1) n}{2}} \tau \sum_{\pi \in \mathfrak{S}_{n}} \operatorname{sgn}(\pi) \\
& \quad \times \sum_{\gamma_{1} \ldots \gamma_{n} \in\{ \pm\}}(-1)^{\gamma_{1}+\cdots+\gamma_{n}}\left(P_{1}^{-\gamma_{1}} \text { ad } f_{\pi(1)} P_{1}^{\gamma_{1}}\right) \cdots\left(P_{n}^{-\gamma_{n}} \operatorname{ad} f_{\pi(n)} P_{n}^{\gamma_{n}}\right) f_{0},
\end{aligned}
$$

where $P_{1}^{+}, \ldots, P_{n}^{+}$is any system of pairwise commuting good idempotents in the sense of Definition 14 (the value does not depend on the choice of the latter). Analogously,

$$
\left({ }^{\otimes} \text { res* }^{*} \varphi\right)\left(f_{1} \wedge \cdots \wedge f_{n}\right)\left(f_{0}\right):=\varphi \cdot{ }^{\otimes} \operatorname{res}_{*}\left(f_{0} \otimes f_{1} \wedge \cdots \wedge f_{n}\right)
$$

for every $\varphi \in k$.

We remark that one can also write the above formula as

$$
\begin{aligned}
& { }^{\otimes} \operatorname{res}_{*}\left(f_{0} \otimes f_{1} \wedge \cdots \wedge f_{n}\right)= \\
& -(-1)^{\frac{(n-1) n}{2}} \tau \sum_{\pi \in \mathfrak{S}_{n}} \operatorname{sgn}(\pi) \sum_{\gamma_{1} \ldots \gamma_{n} \in\{ \pm\}}(-1)^{\gamma_{1}+\cdots+\gamma_{n}}\left(P_{1}^{-\gamma_{1}} f_{\pi(1)} P_{1}^{\gamma_{1}}\right) \cdots\left(P_{n}^{-\gamma_{n}} f_{\pi(n)} P_{n}^{\gamma_{n}}\right) f_{0}
\end{aligned}
$$

since for any expression $g$ we have

$$
\begin{aligned}
P_{i}^{-\gamma_{i}} \operatorname{ad}\left(f_{w}\right) P_{i}^{\gamma_{i}} g & =P_{i}^{-\gamma_{i}}\left[f_{w}, P_{i}^{\gamma_{i}} g\right]=P_{i}^{-\gamma_{i}} f_{w} P_{i}^{\gamma_{i}} g-P_{i}^{-\gamma_{i}} P_{i}^{\gamma_{i}} g f_{w} \\
& =P_{i}^{-\gamma_{i}} f_{w} P_{i}^{\gamma_{i}} g
\end{aligned}
$$

since $P_{i}^{-\gamma_{i}} P_{i}^{\gamma_{i}}=\left(\mathbb{1}-P_{i}^{\gamma_{i}}\right) P_{i}^{\gamma_{i}}=0$ and $P_{i}^{\gamma_{i}}$ is an idempotent.

Proof. Use Proposition 24 with $p=n$. Plugging these components into the shorthand notation of (6-3) we unwind for ${ }^{\otimes} \operatorname{res}_{*}\left(f_{0} \otimes f_{1} \wedge \cdots \wedge f_{n}\right)$ the formula

$$
\begin{aligned}
=-\tau(-1)^{\frac{n^{2}+n}{2}} & \sum_{\substack{w_{1} \ldots w_{n} \\
=\{1, \ldots, n\}}} \rho\left(w_{1}, \ldots, w_{n}\right)(-1)^{w_{1}+\cdots+w_{n}} \\
& \times \sum_{\gamma_{1} \ldots \gamma_{n} \in\{ \pm\}}(-1)^{\gamma_{1}+\cdots+\gamma_{n}}\left(P_{1}^{-\gamma_{1}} \operatorname{ad}\left(f_{w_{n}}\right) P_{1}^{\gamma_{1}}\right) \cdots\left(P_{n}^{-\gamma_{n}} \operatorname{ad}\left(f_{w_{1}}\right) P_{n}^{\gamma_{n}}\right) f_{0} .
\end{aligned}
$$

We can clearly replace $w_{1}, \ldots, w_{n}$ by a sum over all permutations of $\{1, \ldots, n\}$. In order to obtain a nice formula (in the above formula the $P_{i}$ appear in ascending order, while the $w_{i}$ appear in descending order), we prefer to compose each permutation with the order-reversing permutation $w_{i}:=\pi(n-i+1)$; hence, 


$$
\begin{aligned}
& \otimes^{\operatorname{res}_{*}}\left(f_{0} \otimes f_{1} \wedge \cdots \wedge f_{n}\right) \\
&=-\tau(-1)^{\frac{n^{2}+n}{2}} \sum_{\pi \in \mathfrak{S}_{n}} \rho(\pi(n), \ldots, \pi(1))(-1)^{1+\cdots+n} \\
& \quad \times \sum_{\gamma_{1} \ldots \gamma_{n} \in\{ \pm\}}(-1)^{\gamma_{1}+\cdots+\gamma_{n}}\left(P_{1}^{-\gamma_{1}} \operatorname{ad}\left(f_{\pi(1)}\right) P_{1}^{\gamma_{1}}\right) \cdots\left(P_{n}^{-\gamma_{n}} \operatorname{ad}\left(f_{\pi(n)}\right) P_{n}^{\gamma_{n}}\right) f_{0} .
\end{aligned}
$$

To conclude, use (6-6) and the (easy) fact that the order-reversing permutation has sign $(-1)^{(n-1) n / 2}$, giving the sign of our claim.

Proof of Theorems 1 and 2 . We define $\mathfrak{G}:=E^{n}(k)$, where $E$ is the functor defined in Section 1.1. As already discussed in Section 1.1 this contains $k\left[t_{1}^{ \pm}, \ldots, t_{n}^{ \pm}\right]$as a Lie subalgebra, acting as multiplication operators $x \mapsto f \cdot x$. It is also easily checked that the differential operators $t_{1}^{s_{1}} \cdots t_{n}^{s_{n}} \partial_{t_{i}}$ can be written as infinite matrices. If $\mathfrak{g}$ is a finite-dimensional Lie algebra, observe that $\mathfrak{G}=E^{n}(k)$ and $E^{n}\left(\operatorname{End}_{k}(\mathfrak{g})\right)$ are actually isomorphic. If $\mathfrak{g}$ is simple, it is centerless, so the adjoint representation gives an embedding $\mathfrak{g} \hookrightarrow \operatorname{End}_{k}(\mathfrak{g})$, and thus

$$
\mathfrak{g}\left[t_{1}^{ \pm}, \ldots, t_{n}^{ \pm}\right] \hookrightarrow E^{n}\left(\operatorname{End}_{k}(\mathfrak{g})\right) \simeq E^{n}(k)=\mathfrak{G} .
$$

This shows that all Lie algebras in the claim are subalgebras of $\mathfrak{G}$. As shown in Section 1.1, $\mathfrak{G}$ is a cubically decomposed algebra, so we define $\phi$ as in Definition 20, $\phi:=$ res$^{*}(1)$. Since we work with field coefficients, the universal coefficient theorem for Lie algebras tells us that

$$
H^{n+1}(\mathfrak{g}, k) \cong H_{n+1}(\mathfrak{g}, k)^{*},
$$

that is, knowing the values of a cocycle only on Lie cycles (instead of all of $\wedge^{\bullet} \mathfrak{g}$ ) determines the cocycle uniquely, $\operatorname{res}^{*}(1)(\alpha)=\operatorname{res}_{*} \alpha$. However, by Lemma 23 we may evaluate the cocycle on the image of $I$ by using ${ }^{\otimes}$ res $_{*}$ instead. Using

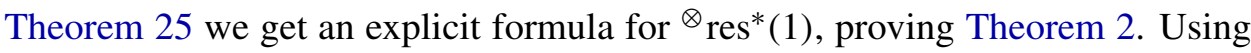
the explicit formula, it is a direct computation to check that for $n=1$ the cocycle agrees with the ones mentioned in the claim of Theorem 1.

\section{Application to the multidimensional residue}

In this section we will show that the Lie cohomology class of Definition 20 naturally gives the multidimensional (Parshin) residue.

We work in the framework of multivariate Laurent polynomial rings over a field $k$; see Section 1.1. In other words, as our cubically decomposed algebra we take an infinite matrix algebra $A=E^{n}(k)$ and $\mathfrak{g}=A_{\text {Lie }}$. Via (1-4) it acts on the $k$-vector space $k\left[t_{1}^{ \pm}, \ldots, t_{n}^{ \pm}\right]$. The latter, now interpreted as a ring, also embeds as a commutative subalgebra into $A$. In order to distinguish very clearly between the subalgebra of $A$ and the vector space it acts on, we shall from now on write 
$k\left[t_{1}^{ \pm}, \ldots, t_{n}^{ \pm}\right]$for the $k$-vector space. Thus, when we write $t_{i}$ we always refer to the associated multiplication operator $x \mapsto t_{i} \cdot x$ in $A$, e.g., $t_{i}^{m} \cdot \boldsymbol{t}_{i}^{l}=\boldsymbol{t}_{i}^{m+l}$.

Following [Beilinson 1980, Lemma 1(b)] we may introduce a (not quite welldefined $^{3}$ ) "map"

$$
\varkappa: \Omega_{k\left[t_{1}^{ \pm}, \ldots, t_{n}^{ \pm}\right] / k}^{n} \rightarrow H_{n+1}(\mathfrak{g}, k), \quad f_{0} \mathrm{~d} f_{1} \wedge \cdots \wedge \mathrm{d} f_{n} \mapsto f_{0} \wedge f_{1} \wedge \cdots \wedge f_{n} .
$$

As $k\left[t_{1}^{ \pm}, \ldots, t_{n}^{ \pm}\right]$is commutative, the $f_{i}$ commute pairwise and thus $f_{0} \wedge \cdots \wedge f_{n}$ is indeed a Lie homology cycle.

Theorem 26. The morphism

$$
\operatorname{res}_{*} \circ \varkappa: \Omega_{k\left[t_{1}^{ \pm}, \ldots, t_{n}^{ \pm}\right] / k}^{n} \rightarrow k
$$

(with $\varkappa$ as in (7-1) and res $_{*}$ as in Definition 20) for $c_{i, j} \in \mathbb{Z}$ is explicitly given by

$t_{1}^{c_{0,1}} \cdots t_{n}^{c_{0, n}} \mathrm{~d}\left(t_{1}^{c_{1,1}} \cdots t_{n}^{c_{1, n}}\right) \wedge \cdots \wedge \mathrm{d}\left(t_{1}^{c_{n, 1}} \cdots t_{n}^{c_{n, n}}\right) \mapsto-(-1)^{\frac{n^{2}+n}{2}} \operatorname{det}\left(\begin{array}{ccc}c_{1,1} & \cdots & c_{n, 1} \\ \vdots & \ddots & \vdots \\ c_{1, n} & \cdots & c_{n, n}\end{array}\right)$

whenever $\sum_{p=0}^{n} c_{p, i}=0$ and is zero otherwise. In particular $-(-1)^{\frac{n^{2}+n}{2}}\left(\operatorname{res}_{*} \circ \varkappa\right)$ is the conventional multidimensional (Parshin) residue.

The complicated sign $-(-1)^{\frac{n^{2}+n}{2}}$ should not concern us too much; it is an artifact of homological algebra. Just by changing our sign conventions for bicomplexes, we could easily switch to an overall opposite sign. Letting $c_{i, j}=\delta_{i=j}$ for $i, j \in$ $\{1, \ldots, n\}$ gives the familiar

$$
-(-1)^{\frac{n^{2}+n}{2}} \operatorname{res}_{*}\left(a t_{1}^{c_{0,1}} \cdots t_{n}^{c_{0, n}} \wedge t_{1} \wedge \cdots \wedge t_{n}\right)=\delta_{c_{0,1}=-1} \cdots \delta_{c_{0, n}=-1} a
$$

for $a \in k$. In particular this assures us that the map res $*$ gives the correct notion of residue: it is the $(-1, \ldots,-1)$-coefficient of the Laurent expansion.

Proof. After unwinding $\varkappa$ it remains to evaluate $\operatorname{res}_{*}\left(f_{0} \wedge f_{1} \wedge \cdots \wedge f_{n}\right)$ for $f_{i}:=t_{1}^{c_{i, 1}} \cdots t_{n}^{c_{i, n}}(i=0, \ldots, n)$. Clearly $f_{0} \otimes f_{1} \wedge \cdots \wedge f_{n}$ is a cycle in $H_{n}(\mathfrak{g}, \mathfrak{g})$, and so by Lemma 23 we may use ${ }^{\otimes}$ res $_{*}$ instead of res $_{*}$. Then Theorem 25 reduces this to the matrix trace

$$
\operatorname{res}_{*}\left(f_{0} \wedge f_{1} \wedge \cdots \wedge f_{n}\right)=-(-1)^{\frac{(n-1) n}{2}} \sum_{\pi \in \mathfrak{S}_{n}} \operatorname{sgn}(\pi) \tau M_{\pi}
$$

${ }^{3}$ It does not respect the relation $\mathrm{d}(a b)=b \mathrm{~d} a+a \mathrm{~d} b$; this artifact already occurs in [Beilinson 1980]. However, this ambiguity dissolves after composing with the residue (as in the theorem) and it is very convenient to treat this as some sort of a map for the moment. 
where

$$
M_{\pi}:=\sum_{\gamma_{1} \ldots \gamma_{n} \in\{ \pm\}}(-1)^{\gamma_{1}+\cdots+\gamma_{n}}\left(P_{1}^{-\gamma_{1}} f_{\pi(1)} P_{1}^{\gamma_{1}}\right) \cdots\left(P_{n}^{-\gamma_{n}} f_{\pi(n)} P_{n}^{\gamma_{n}}\right) f_{0}
$$

For the evaluation of $\tau M_{\pi}$ fix a permutation $\pi$ and pick the (pairwise commuting) system of idempotents given by

$$
P_{j}^{+} \boldsymbol{t}_{1}^{\lambda_{1}} \cdots \boldsymbol{t}_{n}^{\lambda_{n}}=\delta_{\lambda_{j} \geq 0} \boldsymbol{t}_{1}^{\lambda_{1}} \cdots \boldsymbol{t}_{n}^{\lambda_{n}} \quad\left(\text { with } \lambda_{1}, \ldots, \lambda_{n} \in \mathbb{Z}\right) .
$$

Next, observe that the Laurent polynomial ring $W:=k\left[\boldsymbol{t}_{1}^{ \pm}, \ldots, \boldsymbol{t}_{n}^{ \pm}\right]$is stable (i.e., $\phi W \subseteq W$ ) under the endomorphisms $f_{0}, \ldots, f_{n}$ and the idempotents $P_{i}^{ \pm}$, and therefore under $M_{\pi}$. Hence, it follows that it suffices to evaluate the trace of $M_{\pi}$ on the $k$-vector subspace $k\left[\boldsymbol{t}_{1}^{ \pm}, \ldots, \boldsymbol{t}_{n}^{ \pm}\right]$. We compute successively

$$
\begin{gathered}
f_{k} P_{j}^{+} \boldsymbol{t}_{1}^{\lambda_{1}} \cdots \boldsymbol{t}_{n}^{\lambda_{n}}=\delta_{\lambda_{j} \geq 0} \boldsymbol{t}_{1}^{\lambda_{1}+c_{k, 1}} \cdots \boldsymbol{t}_{n}^{\lambda_{n}+c_{k, n}}, \\
P_{j}^{-} f_{k} P_{j}^{+} \boldsymbol{t}_{1}^{\lambda_{1}} \cdots \boldsymbol{t}_{n}^{\lambda_{n}}=\delta_{0 \leq \lambda_{j}<-c_{k, j}} \boldsymbol{t}_{1}^{\lambda_{1}+c_{k, 1}} \cdots \boldsymbol{t}_{n}^{\lambda_{n}+c_{k, n}},
\end{gathered}
$$

and analogously for $P_{j}^{+} f_{k} P_{j}^{-}$. We find

$$
\begin{aligned}
\sum_{\gamma_{j} \in\{ \pm\}}(-1)^{\gamma_{j}}\left(P_{j}^{-\gamma_{j}} f_{k} P_{j}^{\gamma_{j}}\right) & \boldsymbol{t}_{1}^{\lambda_{1}} \cdots \boldsymbol{t}_{n}^{\lambda_{n}} \\
& =\left(\delta_{0 \leq \lambda_{j}<-c_{k, j}}-\delta_{-c_{k, j} \leq \lambda_{j}<0}\right) \boldsymbol{t}_{1}^{\lambda_{1}+c_{k, 1}} \cdots \boldsymbol{t}_{n}^{\lambda_{n}+c_{k, n}} .
\end{aligned}
$$

Subclaim. Writing $w_{i}:=\pi(i)$ we have

$$
\begin{aligned}
& M_{\pi} \boldsymbol{t}_{1}^{\lambda_{1}} \cdots \boldsymbol{t}_{n}^{\lambda_{n}}=\prod_{i=1}^{n}\left(\delta_{0 \leq \lambda_{i}+c_{0, i}+\sum_{p=i+1}^{n} c_{w_{p}, i}<-c_{w_{i}, i}}-\delta_{-c_{w_{i}, i} \leq \lambda_{i}+c_{0, i}+\sum_{p=i+1}^{n} c_{w_{p}, i}<0}\right) \\
& \times \boldsymbol{t}_{1}^{\lambda_{1}+c_{0,1}+\sum_{p=1}^{n} c_{w_{p}, 1}} \cdots \boldsymbol{t}_{n}^{\lambda_{n}+c_{0, n}+\sum_{p=1}^{n} c_{w_{p}, n}} .
\end{aligned}
$$

(Proof of subclaim. Define for $i=1, \ldots, n+1$ the truncated sum

$$
M_{\pi}^{(i)}:=\left[\sum_{\gamma_{i} \ldots \gamma_{n} \in\{ \pm\}}(-1)^{\gamma_{i}+\cdots+\gamma_{n}}\left(P_{i}^{-\gamma_{i}} f_{w_{i}} P_{i}^{\gamma_{i}}\right) \cdots\left(P_{n}^{-\gamma_{n}} f_{w_{n}} P_{n}^{\gamma_{n}}\right)\right] f_{0}
$$

so that $M_{\pi}^{(1)}=M_{\pi}$ and $M_{\pi}^{(n+1)}=f_{0}$. We claim that

$$
M_{\pi}^{(i)} \boldsymbol{t}_{1}^{\lambda_{1}} \cdots \boldsymbol{t}_{n}^{\lambda_{n}}=\alpha \boldsymbol{t}_{1}^{\lambda_{1}+c_{0,1}+\sum_{p=i}^{n} c_{w_{p}, 1}} \cdots \boldsymbol{t}_{n}^{\lambda_{n}+c_{0, n}+\sum_{p=i}^{n} c_{w_{p}, n}}
$$

for some factor $\alpha \in\{ \pm 1,0\}$. For $i=n+1$ this is clear since $f_{0}=t_{1}^{c_{0,1}} \cdots t_{n}^{c_{0, n}}$, in particular $\alpha=1$. Assuming this holds for $i+1$, for $i$ we get by using (7-4) (with 
the appropriate values plugged in: $j:=i$ and $k:=w_{i}$, and $\lambda_{i}$ as in (7-6))

$$
\begin{aligned}
M_{\pi}^{(i)} \boldsymbol{t}_{1}^{\lambda_{1}} \cdots \boldsymbol{t}_{n}^{\lambda_{n}}= & \sum_{\gamma_{i} \in\{ \pm\}}(-1)^{\gamma_{i}}\left(P_{i}^{-\gamma_{i}} f_{w_{i}} P_{i}^{\gamma_{i}}\right) M_{\pi}^{(i+1)} \boldsymbol{t}_{1}^{\lambda_{1}} \cdots \boldsymbol{t}_{n}^{\lambda_{n}} \\
= & \left(\delta_{0 \leq \lambda_{i}+c_{0, i}+\sum_{p=i+1}^{n} c_{w_{p}, i}<-c_{w_{i}, i}}-\delta_{-c_{w_{i}, i} \leq \lambda_{i}+c_{0, i}+\sum_{p=i+1}^{n} c_{w_{p}, i}<0}\right) \\
& \times \alpha \boldsymbol{t}_{1}^{\lambda_{1}+c_{0,1}+\sum_{p=i+1}^{n} c_{w_{p}, 1}+c_{w_{i}, 1}} \cdots \boldsymbol{t}_{n}^{\lambda_{n}+c_{0, n}+\sum_{p=i+1}^{n} c_{w_{p}, n}+c_{w_{i}, n}}
\end{aligned}
$$

This proves our claim for all $i$ by induction. We observe that the prefactor $\alpha$ in each step just gets multiplied with the expression in (7-7), giving the product in our claim.)

Next, we need to evaluate the trace of $M_{\pi}$ as given in (7-5). The endomorphism is nilpotent unless

$$
c_{0,1}+\sum_{p=1}^{n} c_{w_{p}, i}=0 \text { for all } i .
$$

We remark that $w_{1}, \ldots, w_{n}$ is just a permutation of $\{1, \ldots, n\}$, so these conditions can be rewritten as $\sum_{p=0}^{n} c_{p, i}=0$. In the nilpotent case the trace is clearly zero. Hence, we may assume we are in the case where (7-8) holds. Using these equations and the useful convention $w_{n+1}:=0$, our expression for $M_{\pi}$ simplifies to

$$
\begin{aligned}
& M_{\pi} \boldsymbol{t}_{1}^{\lambda_{1}} \cdots \boldsymbol{t}_{n}^{\lambda_{n}} \\
& \quad=\prod_{i=1}^{n}\left(\delta_{0 \leq \lambda_{i}+\sum_{p=i+1}^{n+1} c_{w_{p}, i}<-c_{w_{i}, i}}-\delta_{0 \leq \lambda_{i}+c_{w_{i}, i}+\sum_{p=i+1}^{n+1} c_{w_{p}, i}<c_{w_{i}, i}}\right) \boldsymbol{t}_{1}^{\lambda_{1}} \cdots \boldsymbol{t}_{n}^{\lambda_{n}} .
\end{aligned}
$$

The endomorphism $M_{\pi}$ is visibly diagonal of finite rank and we may reduce the computation of the trace to a (finite-dimensional) stable vector subspace. A finite subset of the $\boldsymbol{t}_{1}^{\lambda_{1}} \cdots \boldsymbol{t}_{n}^{\lambda_{n}}\left(\lambda_{1}, \ldots, \lambda_{n} \in \mathbb{Z}\right)$ provides a basis. We see in (7-9) that $M_{\pi}$ acts diagonally on these basis vectors with eigenvalues \pm 1 or 0 . Moreover, for each $i$ we either have $c_{w_{i}, i} \geq 0$ or $c_{w_{i}, i}<0$, which shows that each bracket of the shape $\left(\delta_{0 \leq \lambda<-c}-\delta_{-c \leq \lambda<0}\right)$ in (7-9) either attains only values in $\{+1,0\}$ when we run through all $\lambda_{1}, \ldots, \lambda_{n} \in \mathbb{Z}$, or only values in $\{-1,0\}$. This shows that we only need to count (with appropriate sign) the nonzero eigenvalues of $M_{\pi}$ in order to evaluate the trace. Note that our finite subset of $\boldsymbol{t}_{1}^{\lambda_{1}} \cdots \boldsymbol{t}_{n}^{\lambda_{n}}\left(\lambda_{1}, \ldots, \lambda_{n} \in \mathbb{Z}\right)$ indexes a basis, so we need to count the number of such basis vectors with nonzero eigenvalue. We introduce the nonstandard shorthand $\lfloor x\rfloor:=\min (0, x)$. Inspecting (7-9) shows that when running through $\lambda_{i}$ we have

- $\left\lfloor-c_{w_{i}, i}\right\rfloor$ times the eigenvalue +1 ,

- $\left\lfloor+c_{w_{i}, i}\right\rfloor$ times the eigenvalue -1 . 
The value of a fixed bracket $\left(\delta_{0 \leq \lambda<-c}-\delta_{-c \leq \lambda<0}\right)$ - when nonzero - is always either +1 , or always -1 . Thus, the number of nonzero eigenvalues is simply the number of elements within the hypercube such that each $\lambda_{i}$ lies within the range of length $\left\lfloor \pm c_{w_{i}, i}\right\rfloor$ counted above, and therefore

$$
\tau M_{\pi}=\prod_{i=1}^{n}\left(\left\lfloor-c_{w_{i}, i}\right\rfloor-\left\lfloor+c_{w_{i}, i}\right\rfloor\right)=\prod_{i=1}^{n}\left(-c_{w_{i}, i}\right)=(-1)^{n} \prod_{i=1}^{n} c_{\pi(i), i}
$$

(because $\lfloor-a\rfloor-\lfloor a\rfloor=-a$ for all $a \in \mathbb{Z}$ ). We plug this into (7-2) and recognize the usual formula for the determinant. This finishes the proof.

We are now ready to prove the remaining theorems from the introduction:

Proof of Theorems 4 and 5. We use Theorem 26 to obtain Theorem 4(2). Then Theorem 4(3) follows as a special case. For Theorem 4(1) use the shorthands $\pi=P_{1}^{+}=P^{+}$(following both the notation of Arbarello, de Concini and Kac and ours). On the one hand we compute

$$
\begin{aligned}
{\left[\pi, f_{1}\right] f_{0} } & =\left[P, f_{1}\right] f_{0}=P f_{1} f_{0}-f_{1} P f_{0}=\left[P f_{0}, f_{1}\right] \\
& =\left(P^{+}+P^{-}\right)\left[P^{+} f_{0}, f_{1}\right] \\
& =P^{-}\left[P^{+} f_{0}, f_{1}\right]+P^{+}\left[P^{+} f_{0}, f_{1}\right] \\
& =P^{-}\left[P^{+} f_{0}, f_{1}\right]-P^{+}\left[P^{-} f_{0}, f_{1}\right] .
\end{aligned}
$$

where for the last equality we used that $\left[P^{+} f_{0}, f_{1}\right]+\left[P^{-} f_{0}, f_{1}\right]=\left[f_{0}, f_{1}\right]=0$. On the other hand, we unwind

$$
\text { res } \begin{aligned}
f_{0} \mathrm{~d} f_{1} & =(-1)^{1} \operatorname{tr} \sum_{\gamma_{1} \in\{ \pm\}}(-1)^{\gamma_{1}}\left(P_{1}^{-\gamma_{1}} \operatorname{ad}\left(f_{\pi(1)}\right) P_{1}^{\gamma_{1}}\right) f_{0} \\
& =-P^{-}\left[f_{1}, P_{1}^{+} f_{0}\right]+P^{+}\left[f_{1}, P_{1}^{-} f_{0}\right]
\end{aligned}
$$

and these expressions clearly coincide. Finally Theorem 5 is true since we use the cocycle defined in Definition 20, which is constructed exactly as stated in Theorem 5.

\section{Application to multiloop Lie algebras}

Suppose $k$ is a field and $\mathfrak{g} / k$ is a finite-dimensional centerless Lie algebra (e.g., $\mathfrak{g}$ finite-dimensional, semisimple). Then the adjoint representation ad: $\mathfrak{g} \hookrightarrow \operatorname{End}_{k}(\mathfrak{g})$ is injective. Thus, we obtain a Lie algebra inclusion

$$
i: \mathfrak{g}\left[\boldsymbol{t}_{1}^{ \pm}, \ldots, \boldsymbol{t}_{n}^{ \pm}\right] \hookrightarrow E^{n}\left(\operatorname{End}_{k}(\mathfrak{g})\right)_{\text {Lie }},
$$

where $E$ is the functor described in Section 1.1 (the right-hand side is equipped with the Lie bracket $[a, b]=a b-b a$ based on the associative algebra structure). 
Thus, we have the pullback

$$
i^{*}: H^{n+1}\left(E^{n}\left(\operatorname{End}_{R}(\mathfrak{g})\right)_{\text {Lie }}, k\right) \rightarrow H^{n+1}\left(\mathfrak{g}\left[\boldsymbol{t}_{1}^{ \pm}, \ldots, \boldsymbol{t}_{n}^{ \pm}\right], k\right),
$$

which we may apply to the class res*(1) - see Definition 20.

Theorem 27. Suppose $k$ is a field and $\mathfrak{g} / k$ is a finite-dimensional centerless Lie algebra. For $Y_{0}, \ldots, Y_{n} \in \mathfrak{g}$ we call

$$
B\left(Y_{0}, \ldots, Y_{n}\right):=\operatorname{tr}_{\operatorname{End}_{k}(\mathfrak{g})}\left(\operatorname{ad}\left(Y_{0}\right) \operatorname{ad}\left(Y_{1}\right) \cdots \operatorname{ad}\left(Y_{n}\right)\right)
$$

the "generalized Killing form". For $n=1$ and if $\mathfrak{g}$ is semisimple, this is the classical Killing form of $\mathfrak{g}$.

(1) Then on all Lie cycles admitting a lift under I as in (0-1), the pullback $i^{*} \operatorname{res}^{*}(1) \in H^{n+1}\left(\mathfrak{g}\left[\boldsymbol{t}_{1}^{ \pm}, \ldots, \boldsymbol{t}_{n}^{ \pm}\right], k\right)$ is explicitly given by

$$
\begin{aligned}
\left(i^{*} \phi\right)\left(Y_{0} t_{1}^{c_{0,1}} \cdots \boldsymbol{t}_{n}^{c_{0, n}}\right. & \left.\wedge \cdots \wedge Y_{n} \boldsymbol{t}_{1}^{c_{n, 1}} \cdots \boldsymbol{t}_{n}^{c_{n, n}}\right) \\
= & -(-1)^{\frac{n^{2}+n}{2}} \sum_{\pi \in \mathfrak{S}_{n}} \operatorname{sgn}(\pi) B\left(Y_{\pi(1)}, \ldots, Y_{\pi(n)}, Y_{0}\right) \prod_{i=1}^{n} c_{\pi(i), i}
\end{aligned}
$$

whenever $\sum_{p=0}^{n} c_{p, i}=0$ for all $i \in\{1, \ldots, n\}$, and it vanishes otherwise.

(2) If $\mathfrak{g}$ is finite-dimensional and semisimple and $n=1$, then $i^{*} \operatorname{res}^{*}(1) \in H^{2}\left(\mathfrak{g}\left[\boldsymbol{t}_{1}^{ \pm}\right], k\right)$ is the universal central extension of the loop Lie algebra $\mathfrak{g}\left[\boldsymbol{t}_{1}, \boldsymbol{t}_{1}^{-1}\right]$ giving the associated affine Lie algebra $\widehat{\mathfrak{g}}$ (without extending by a derivation),

$$
0 \rightarrow k\langle c\rangle \rightarrow \widehat{\mathfrak{g}} \rightarrow \mathfrak{g}\left[\boldsymbol{t}_{1}, \boldsymbol{t}_{1}^{-1}\right] \rightarrow 0 .
$$

Proof. (1) By Lemma 23, Theorem 25 and (5-2) the cocycle is explicitly given by

$$
\operatorname{res}^{*}(1)\left(f_{0} \wedge \cdots \wedge f_{n}\right)={ }^{\otimes} \operatorname{res}^{*}(1)\left(f_{0} \otimes f_{1} \wedge \cdots \wedge f_{n}\right)=\tau \sum_{\pi \in \mathfrak{S}_{n}} \operatorname{sgn}(\pi) M_{\pi},
$$

where

$$
M_{\pi}=\sum_{\gamma_{1} \ldots \gamma_{n} \in\{ \pm\}}(-1)^{\gamma_{1}+\cdots+\gamma_{n}}\left(P_{1}^{-\gamma_{1}} f_{\pi(1)} P_{1}^{\gamma_{1}}\right) \cdots\left(P_{n}^{-\gamma_{n}} f_{\pi(n)} P_{n}^{\gamma_{n}}\right) f_{0} .
$$

Note that $M_{\pi} \in E^{n}\left(\operatorname{End}_{k}(\mathfrak{g})\right)$. As we consider the pullback of the cohomology class along $i: \mathfrak{g}\left[t_{1}^{ \pm}, \ldots, t_{n}^{ \pm}\right] \hookrightarrow E^{n}\left(\operatorname{End}_{k}(\mathfrak{g})\right)_{\text {Lie }}$, it suffices to treat elements $f_{i}:=Y_{i} t_{1}^{c_{i, 1}} \cdots t_{n}^{c_{i, n}}$ with $c_{i, 1}, \ldots, c_{i, n} \in \mathbb{Z}$ (for $i=0, \ldots, n$ ) and $Y_{i} \in \mathfrak{g}$. Note that by our embedding $i$ an element $f_{i}$ is mapped to the endomorphism $\operatorname{ad}\left(Y_{i}\right) t_{1}^{c_{i, 1}} \cdots t_{n}^{c_{i, n}}$ in $E^{n}\left(\operatorname{End}_{k}(\mathfrak{g})\right)$. Let $\pi \in \mathfrak{S}_{n}$ be a fixed permutation. In order to compute the trace, it suffices to study the action of $M_{\pi}$ on the basis elements $X t_{1}^{\lambda_{1}} \cdots t_{n}^{\lambda_{n}}$ of $\mathfrak{g}\left[t_{1}^{ \pm}, \ldots, t_{n}^{ \pm}\right]$, where $\lambda_{1}, \ldots, \lambda_{n} \in \mathbb{Z}$ and $X \in \mathfrak{g}$ runs through a basis of $\mathfrak{g}$. We denote them with bold letters $\boldsymbol{t}_{i}$ instead of $t_{i}$ to distinguish clearly between a basis element and $t_{i}$ as an endomorphism $t_{i}: x \mapsto t_{i} \cdot x$ in $E^{n}\left(\operatorname{End}_{k}(\mathfrak{g})\right)$. As in the proof 
of Theorem 26 we compute

$$
P_{j}^{-} f_{k} P_{j}^{+} X \boldsymbol{t}_{1}^{\lambda_{1}} \cdots \boldsymbol{t}_{n}^{\lambda_{n}}=\delta_{0 \leq \lambda_{j}<-c_{k, j}} \operatorname{ad}\left(Y_{k}\right) X \boldsymbol{t}_{1}^{\lambda_{1}+c_{k, 1}} \cdots \boldsymbol{t}_{n}^{\lambda_{n}+c_{k, n}},
$$

and as a consequence we find

$$
\begin{aligned}
\sum_{\gamma_{j} \in\{ \pm\}}(-1)^{\gamma_{j}}\left(P_{j}^{-\gamma_{j}} f_{k} P_{j}^{\gamma_{j}}\right) X \boldsymbol{t}_{1}^{\lambda_{1}} \cdots \boldsymbol{t}_{n}^{\lambda_{n}} \\
=\left(\delta_{0 \leq \lambda_{j}<-c_{k, j}}-\delta_{-c_{k, j} \leq \lambda_{j}<0}\right) \operatorname{ad}\left(Y_{k}\right) X \boldsymbol{t}_{1}^{\lambda_{1}+c_{k, 1}} \cdots \boldsymbol{t}_{n}^{\lambda_{n}+c_{k, n}} .
\end{aligned}
$$

With an inductive computation entirely analogous to (7-5) we find

$$
\begin{aligned}
M_{\pi} X \boldsymbol{t}_{1}^{\lambda_{1}} \cdots \boldsymbol{t}_{n}^{\lambda_{n}}=\prod_{i=1}^{n} & \left(\delta_{0 \leq \lambda_{i}+c_{0, i}+\sum_{p=i+1}^{n} c_{w_{p}, i}<-c_{w_{i}, i}}-\delta_{-c_{w_{i}, i} \leq \lambda_{i}+c_{0, i}+\sum_{p=i+1}^{n} c_{w_{p}, i}<0}\right) \\
& \times \operatorname{ad}\left(Y_{w_{1}}\right) \cdots \operatorname{ad}\left(Y_{w_{n}}\right) \operatorname{ad}\left(Y_{0}\right) X \boldsymbol{t}_{1}^{\lambda_{1}+\sum_{p=0}^{n} c_{p, 1}} \cdots \boldsymbol{t}_{n}^{\lambda_{n}+\sum_{p=0}^{n} c_{p, n}},
\end{aligned}
$$

where $w_{i}:=\pi(i)$. Unless $\forall i: \sum_{p=0}^{n} c_{p, i}=0$ holds, $M_{\pi}$ is clearly nilpotent and thus has trace $\tau M_{\pi}=0$. This condition is clearly independent of $\pi$, showing that $\left(i^{*} \operatorname{res}^{*}(1)\right)\left(f_{0} \wedge \cdots \wedge f_{n}\right)=0$ in this case. From now on assume $\forall i: \sum_{p=0}^{n} c_{p, i}=0$. Then $M_{\pi}$ respects the decomposition

$$
\mathfrak{g}\left[\boldsymbol{t}_{1}^{ \pm}, \ldots, \boldsymbol{t}_{n}^{ \pm}\right]=\coprod_{\lambda_{1} \ldots \lambda_{n} \in \mathbb{Z}^{n}} \mathfrak{g} t_{1}^{\lambda_{1}} \cdots \boldsymbol{t}_{n}^{\lambda_{n}}
$$

and therefore (as $\tau$ is essentially a trace) $\tau M_{\pi}=\left.\sum_{\lambda_{1}, \ldots, \lambda_{n}} \tau M_{\pi}\right|_{\mathfrak{g} t_{1}^{\lambda_{1}} \ldots t_{n}^{\lambda_{n}}}$. For each summand of the latter we obtain

$$
\begin{aligned}
\left.\tau M_{\pi}\right|_{\mathfrak{g} t_{1}^{\lambda_{1}} \ldots t_{n}^{\lambda_{n}}}=\prod_{i=1}^{n}\left(\delta_{0 \leq \lambda_{i}+c_{0, i}+\sum_{p=i+1}^{n} c_{w_{p}, i}<-c_{w_{i}, i}}\right. & \left.-\delta_{-c_{w_{i}, i} \leq \lambda_{i}+c_{0, i}+\sum_{p=i+1}^{n} c_{w_{p}, i}<0}\right) \\
& \times \operatorname{tr}\left(\operatorname{ad}\left(Y_{w_{1}}\right) \cdots \operatorname{ad}\left(Y_{w_{n}}\right) \operatorname{ad}\left(Y_{0}\right)\right) .
\end{aligned}
$$

The trace term is independent of $\lambda_{1}, \ldots, \lambda_{n}$ (and in the shape of (8-1)), so we may rewrite $\tau M_{\pi}$ as

$$
\begin{aligned}
& \tau M_{\pi}= B\left(Y_{w_{1}}, \ldots, Y_{w_{n}}, Y_{0}\right) \\
& \times \sum_{\lambda_{1}, \ldots, \lambda_{n}} \prod_{i=1}^{n}\left(\delta_{0 \leq \lambda_{i}+c_{0, i}}+\sum_{p=i+1}^{n} c_{w_{p}, i}<-c_{w_{i}, i}\right. \\
&\left.-\delta_{-c_{w_{i}, i}<\lambda_{i}+c_{0, i}+\sum_{p=i+1}^{n} c_{w_{p}, i}<0}\right) .
\end{aligned}
$$

For the evaluation of the sum $\sum_{\lambda_{1}, \ldots, \lambda_{n}}$ we can apply the same eigenvalue count as in the proof of Theorem 26. This time instead of counting eigenvalues, we count nonzero summands. This yields

$$
\tau M_{\pi}=(-1)^{n} B\left(Y_{w_{1}}, \ldots, Y_{w_{n}}, Y_{0}\right) \prod_{i=1}^{n} c_{w_{i}, i}
$$

and thus our claim. 
(2) For $n=1$ we obtain

$$
\left(i^{*} \operatorname{res}^{*}(1)\right)\left(Y_{0} t_{1}^{c_{0,1}} \wedge Y_{1} t_{1}^{c_{1,1}}\right)=-c_{1,1} \delta_{c_{0,1}+c_{1,1}=0} B\left(Y_{1}, Y_{0}\right) .
$$

This is well-known to be the defining cocycle of the affine Lie algebra $\widehat{\mathfrak{g}}$ (usually with a positive sign, but the class is only well-defined up to nonzero scalar multiple anyway).

The natural further cases of the Virasoro algebra as well as affine Kac-Moody algebras (i.e., $\widehat{\mathfrak{g}}$ extended by derivations) will be discussed elsewhere. The computations become more involved, but no further ideas are needed.

\section{Acknowledgements}

I am very thankful to Ivan Fesenko and Matthew Morrow for many valuable discussions, especially on an adèle interpretation. I thank the research group of Professor Marc Levine for the stimulating scientific environment. I heartily thank the anonymous referee for greatly improving the presentation, especially in Section 4, and observing the fact $H^{2}=0$ in (4-4), which clarifies a crucial cancellation in the proof of Proposition 24.

\section{References}

[Arbarello et al. 1989] E. Arbarello, C. De Concini, and V. G. Kac, "The infinite wedge representation and the reciprocity law for algebraic curves", pp. 171-190 in Theta functions, Part 1 (Bowdoin College, Brunswick, ME, 1987), edited by L. Ehrenpreis and R. C. Gunning, Proc. Sympos. Pure Math. 49, Amer. Math. Soc., Providence, RI, 1989. MR 90i:22034 Zbl 0699.22028

[Beilinson 1980] A. A. Beilinson, "Residues and adèles", Funktsional. Anal. i Prilozhen. 14:1 (1980), 44-45. MR 81f:14010 Zbl 0509.14018

[Beilinson et al. 1991] A. A. Beilinson, B. L. Feigin, and B. C. Mazur, "Notes on conformal field theory", unpublished, 1991, Available at http://www.math.sunysb.edu/ kirillov/manuscripts.html.

[Fer̆gin and Tsygan 1983] B. L. Fer̆gin and B. L. Tsygan, "Cohomology of Lie algebras of generalized Jacobi matrices”, Funktsional. Anal. i Prilozhen. 17:2 (1983), 86-87. MR 85c:17008 Zbl 0544.17011

[Fesenko 2010] I. Fesenko, "Analysis on arithmetic schemes, II", J. K-Theory 5:3 (2010), 437-557. MR 2011k:14019 Zbl 1225.14019

[Fesenko and Kurihara 2000] I. Fesenko and M. Kurihara (editors), Invitation to higher local fields, Geometry \& topology monographs 3, Geometry \& topology, Coventry, England, 2000. Papers from the conference held in Münster, August 29-September 5, 1999,. MR 2001h:11005 Zbl 0954.00026

[Frenkel 1987] I. B. Frenkel, "Beyond affine Lie algebras", pp. 821-839 in Proceedings of the International Congress of Mathematicians (Berkeley, CA, 1986), vol. 1, 2, edited by A. M. Gleason, Amer. Math. Soc., Providence, RI, 1987. MR 89g:17018 Zbl 0668.17016

[Frenkel and Zhu 2012] E. Frenkel and X. Zhu, "Gerbal representations of double loop groups", Int. Math. Res. Not. 2012:17 (2012), 3929-4013. MR 2972546 Zbl 06088719

[Huber 1991] A. Huber, "On the Parshin-Běllinson adèles for schemes", Abh. Math. Sem. Univ. Hamburg 61 (1991), 249-273. MR 92k:14024 Zbl 0763.14006 
[Hübl and Yekutieli 1996] R. Hübl and A. Yekutieli, "Adèles and differential forms", J. Reine Angew. Math. 471 (1996), 1-22. MR 97d:14026 Zbl 0847.14006

[Jimbo and Miwa 1983] M. Jimbo and T. Miwa, "Solitons and infinite-dimensional Lie algebras", Publ. Res. Inst. Math. Sci. 19:3 (1983), 943-1001. MR 85i:58060 Zbl 0557.35091

[Kac and Peterson 1981] V. G. Kac and D. H. Peterson, "Spin and wedge representations of infinitedimensional Lie algebras and groups", Proc. Nat. Acad. Sci. U.S.A. 78:6, part 1 (1981), 3308-3312. MR 82j:17019 Zbl 0469.22016

[Kac and Raina 1987] V. G. Kac and A. K. Raina, Bombay lectures on highest weight representations of infinite-dimensional Lie algebras, Advanced series in mathematical physics 2, World scientific, Teaneck, NJ, 1987. MR 90k:17013 Zbl 0668.17012

[Loday 1992] J.-L. Loday, Cyclic homology, Grundlehren der Mathematischen Wissenschaften [Fundamental principles of mathematical sciences] 301, Springer, Berlin, 1992. MR 94a:19004 Zbl 0780.18009

[Morrow 2010] M. Morrow, "An explicit approach to residues on and dualizing sheaves of arithmetic surfaces”, New York J. Math. 16 (2010), 575-627. MR 2012a:14061 Zbl 1258.14031

[Neher 2011] E. Neher, "Extended affine Lie algebras and other generalizations of affine Lie algebrasa survey", pp. 53-126 in Developments and trends in infinite-dimensional Lie theory, edited by K.-H. Neeb and A. Pianzola, Progr. Math. 288, Birkhäuser, Boston, 2011. MR 2011m:17055 Zbl 1261.17023

[Tate 1968] J. Tate, "Residues of differentials on curves", Ann. Sci. École Norm. Sup. (4) 1 (1968), 149-159. MR 37 \#2756 Zbl 0159.22702

Communicated by Mikhail Kapranov

Received 2012-06-16 Revised 2013-04-14 Accepted 2013-09-09

oliver.braeunling@uni-due.de Fakultät für Mathematik, Universität Duisburg-Essen, Thea-Leymann-Straße 9, 45127 Essen, Germany http://www.esaga.uni-due.de/oliver.braeunling/ 


\section{Algebra \& Number Theory}

msp.org/ant

\section{EDITORS}

MANAGING EDITOR

Bjorn Poonen

Massachusetts Institute of Technology

Cambridge, USA

\author{
EDITORIAL BOARD CHAIR \\ David Eisenbud \\ University of California \\ Berkeley, USA
}

\section{BOARD OF EDITORS}

Georgia Benkart

Dave Benson

Richard E. Borcherds

John H. Coates

J-L. Colliot-Thélène

Brian D. Conrad

Hélène Esnault

Hubert Flenner

Edward Frenkel

Andrew Granville

Joseph Gubeladze

Roger Heath-Brown

Ehud Hrushovski

Craig Huneke

Mikhail Kapranov

Yujiro Kawamata

János Kollár

Yuri Manin

Barry Mazur

Philippe Michel
University of Wisconsin, Madison, USA

University of Aberdeen, Scotland

University of California, Berkeley, USA

University of Cambridge, UK

CNRS, Université Paris-Sud, France

University of Michigan, USA

Freie Universität Berlin, Germany

Ruhr-Universität, Germany

University of California, Berkeley, USA

Université de Montréal, Canada

San Francisco State University, USA

Oxford University, UK

Hebrew University, Israel

University of Virginia, USA

Yale University, USA

University of Tokyo, Japan

Princeton University, USA

Northwestern University, USA

Harvard University, USA

École Polytechnique Fédérale de Lausanne
Susan Montgomery

Shigefumi Mori

Raman Parimala

Jonathan Pila

Victor Reiner

Karl Rubin

Peter Sarnak

Joseph H. Silverman

Michael Singer

Vasudevan Srinivas

J. Toby Stafford

Bernd Sturmfels

Richard Taylor

Ravi Vakil

Michel van den Bergh

Marie-France Vignéras

Kei-Ichi Watanabe

Efim Zelmanov

Shou-Wu Zhang
University of Southern California, USA

RIMS, Kyoto University, Japan

Emory University, USA

University of Oxford, UK

University of Minnesota, USA

University of California, Irvine, USA

Princeton University, USA

Brown University, USA

North Carolina State University, USA

Tata Inst. of Fund. Research, India

University of Michigan, USA

University of California, Berkeley, USA

Harvard University, USA

Stanford University, USA

Hasselt University, Belgium

Université Paris VII, France

Nihon University, Japan

University of California, San Diego, USA

Princeton University, USA

PRODUCTION

production@msp.org

Silvio Levy, Scientific Editor

See inside back cover or msp.org/ant for submission instructions.

The subscription price for 2014 is US $\$ 225 /$ year for the electronic version, and $\$ 400 /$ year $(+\$ 55$, if shipping outside the US) for print and electronic. Subscriptions, requests for back issues and changes of subscribers address should be sent to MSP.

Algebra \& Number Theory (ISSN 1944-7833 electronic, 1937-0652 printed) at Mathematical Sciences Publishers, 798 Evans Hall \#3840, c/o University of California, Berkeley, CA 94720-3840 is published continuously online. Periodical rate postage paid at Berkeley, CA 94704, and additional mailing offices.

ANT peer review and production are managed by EditFLOW ${ }^{\circledR}$ from Mathematical Sciences Publishers.

\section{PUBLISHED BY}

- mathematical sciences publishers

nonprofit scientific publishing

http://msp.org/

(C) 2014 Mathematical Sciences Publishers 


\section{Algebra \& Number Theory}

\section{Volume $8 \quad$ No. $1 \quad 2014$}

On the Picard number of K3 surfaces over number fields

FRANÇOIS CHARLES

Adèle residue symbol and Tate's central extension for multiloop Lie algebras

OLIVER BRAUNLING

On the number of cubic orders of bounded discriminant having automorphism group $C_{3}$, and related problems

MANJUl Bhargava and ARIEl ShNidman

Polynomial bounds for Arakelov invariants of Belyi curves

ARIYAN JAVANPEYKAR

The radius of a subcategory of modules

HAILONG DAO and RYO TAKAHASHI

A generalized Bogomolov-Gieseker inequality for the three-dimensional projective space

EMANUELE MACRÌ

$(\varphi, \Gamma)$-modules over noncommutative overconvergent and Robba rings

GERGELY ZÁBRÁDI

The Tannakian formalism and the Langlands conjectures

DAVID KAZHDAN, MICHAEL LARSEN and YAKOV VARSHAVSKY 